\title{
RESEARCH
}

Open Access

\section{High throughput sequencing provides exact genomic locations of inducible prophages and accurate phage-to-host ratios in gut microbial strains}

Mirjam Zünd ${ }^{1}$, Hans-Joachim Ruscheweyh ${ }^{1}$, Christopher M. Field ${ }^{1}$, Natalie Meyer ${ }^{1}$, Miguelangel Cuenca', Daniel Hoces ${ }^{2}$, Wolf-Dietrich Hardt ${ }^{1}$ and Shinichi Sunagawa ${ }^{1 *}$ (D)

\begin{abstract}
Background: Temperate phages influence the density, diversity and function of bacterial populations. Historically, they have been described as carriers of toxins. More recently, they have also been recognised as direct modulators of the gut microbiome, and indirectly of host health and disease. Despite recent advances in studying prophages using non-targeted sequencing approaches, methodological challenges in identifying inducible prophages in bacterial genomes and quantifying their activity have limited our understanding of prophage-host interactions.

Results: We present methods for using high-throughput sequencing data to locate inducible prophages, including those previously undiscovered, to quantify prophage activity and to investigate their replication. We first used the well-established Salmonella enterica serovar Typhimurium/p22 system to validate our methods for (i) quantifying phage-to-host ratios and (ii) accurately locating inducible prophages in the reference genome based on phage-tohost ratio differences and read alignment alterations between induced and non-induced prophages. Investigating prophages in bacterial strains from a murine gut model microbiota known as Oligo-MM ${ }^{12}$ or sDMDMm2, we located five novel inducible prophages in three strains, quantified their activity and showed signatures of lateral transduction potential for two of them. Furthermore, we show that the methods were also applicable to metagenomes of induced faecal samples from Oligo-MM ${ }^{12}$ mice, including for strains with a relative abundance below 1\%, illustrating its potential for the discovery of inducible prophages also in more complex metagenomes. Finally, we show that predictions of prophage locations in reference genomes of the strains we studied were variable and inconsistent for four bioinformatic tools we tested, which highlights the importance of their experimental validation.

\footnotetext{
* Correspondence: ssunagawa@ethz.ch

'Department of Biology, Institute of Microbiology and Swiss Institute of Bioinformatics, ETH Zürich, Zürich, Switzerland

Full list of author information is available at the end of the article
}

C C The Author(s). 2021 Open Access This article is licensed under a Creative Commons Attribution 4.0 International License, which permits use, sharing, adaptation, distribution and reproduction in any medium or format, as long as you give appropriate credit to the original author(s) and the source, provide a link to the Creative Commons licence, and indicate if changes were made. The images or other third party material in this article are included in the article's Creative Commons licence, unless indicated otherwise in a credit line to the material. If material is not included in the article's Creative Commons licence and your intended use is not permitted by statutory regulation or exceeds the permitted use, you will need to obtain permission directly from the copyright holder. To view a copy of this licence, visit http://creativecommons.org/licenses/by/4.0/ The Creative Commons Public Domain Dedication waiver (http://creativecommons.org/publicdomain/zero/1.0/) applies to the data made available in this article, unless otherwise stated in a credit line to the data. 


\begin{abstract}
(Continued from previous page)
Conclusions: This study demonstrates that the integration of experimental induction and bioinformatic analysis presented here is a powerful approach to accurately locate inducible prophages using high-throughput sequencing data and to quantify their activity. The ability to generate such quantitative information will be critical in helping us to gain better insights into the factors that determine phage activity and how prophage-bacteria interactions influence our microbiome and impact human health.
\end{abstract}

Keywords: Prophage localisation, Prophage induction, Phage activity, Phage replication, High-throughput sequencing, Phage-to-host ratio, Gut microbiota

\section{Background}

The gut microbiome of humans and other mammals is composed of a diverse range of eukaryotic microorganisms, archaea, bacteria and viruses [1]. Compared to the different types of microorganisms identified in the gut, viruses that infect bacteria (phages) are suggested to be numerically dominant, highly diverse, individual-specific and stable over time [2-5]. Of the gut phages that have been identified to date, many display a 'temperate', lysogenic life cycle in which they replicate as either genomeintegrated or extrachromosomal prophages within host bacterial cells [6]. Upon induction, temperate phages can enter the lytic cycle, during which they replicate within the host cell and produce new infective particles (virions) that are released upon lysis of the host cell [7].

The widespread manifestation of lysogeny amongst phages found in the gut microbiome suggests an important role for prophages in controlling the density, diversity and function of gut bacterial populations [8-10]. Prophages can be induced to enter the lytic cycle either spontaneously [11], or by stress stimuli [12-15]. The subsequent production and release of virions is usually lethal to bacterial host cells, and may also impact nonsusceptible species within a gut bacterial community through cascading effects that owe to the disturbance caused to bacterial interaction networks [16]. The activity and abundance of phages may thus cause substantial alterations to gut microbial community compositions [17] and in turn, impact host-microbe interactions. Indeed, phages are increasingly recognised to have an essential role in the health and disease of mammals. Alteration in the abundance and composition of viral populations have been detected, for example, in the context of gastrointestinal disorders [18-20]; after dietary perturbation in humans [21] and mice [22, 23], as well as in response to antibiotic administration in pigs [14].

Whilst the importance of prophages as mobile genetic elements [24] and modulators of the gut microbiome [8] is now evident, methodological challenges in identifying prophages in bacterial genomes and in assessing their inducibility still limit our understanding of prophage-host interactions. For example, studying prophages can be laborious when relying on targeted cultivation- and imaging-based approaches $[25,26]$. As an alternative, recent advances have enabled researchers to identify and characterise phages by analysing high-throughput sequencing (HTS) data, including in uncultured gut microbial community (i.e. faecal) samples $[2,27]$. One approach is to enrich virions from cultures or communities of host bacteria, extract the DNA of viral genomes and subject it to shotgun metagenomic sequencing. This approach makes it possible to reconstruct entire viral genomes from shotgun sequence data, has led to increased expansion of viral genome sequences in public databases [28] and provided an improved ability to identify active prophage producing virions [29]. However, the choice of enrichment methods was shown to influence the number of recovered virus types [30] and the low yield of DNA isolated from virions usually necessitates an additional amplification step, which is prone to generating uneven or chimeric representations of genomic fragments [31, 32]. Together, the pre-treatment steps before virion sequencing thus commonly lead to biases in the relative abundance estimation of viruses.

To overcome the limitation of low DNA yields from enriched virions, an alternative approach is to extract DNA from whole cultures or community samples without an enrichment step and shotgun sequence the entire community. Using this approach, the activity of prophages in the entire culture can be quantified by assessing the relative copy number of phage genomes per host genome-known as the phage to host $(\mathrm{PtoH})$ ratio. For example, using HTS data from human gut microbiome samples, Waller et al. (2014) sought to quantify $\mathrm{PtoH}$ ratios by aligning sequencing reads to prophage regions in sequenced reference genomes [33]. This approach relies on the use of bioinformatics tools, such as VirSorter [34], VIBRANT [35], PHASTER [36] and Prophage Hunter [37], which predict prophage regions in fragmented or complete microbial genomes by using information from virus databases and testing for the presence of viral hallmark genes [38]. However, these methods rely on similarity searches within existing databases or machine learning using known phage sequences for training. This may bias the prediction of novel prophages in favour of those that are most similar to 
previously reported prophages. Furthermore, to our knowledge, no attempts have been made to assess the accuracy of different tools in predicting the genomic coordinates (i.e. locations) of prophages and to experimentally validate whether HTS-derived PtoH ratios are in congruence with established quantitative methods, such as quantitative PCR (qPCR).

Here, we describe the development and validation of methods (i) to test the inducibility, (ii) to determine the precise genomic location and (iii) to explore the replication mode of prophages, and (iv) to accurately quantify $\mathrm{PtoH}$ ratios. We subsequently applied these methods to identify, locate and characterise several inducible prophages in gut microbial model strains. We further show that this approach can also be used to analyse prophage activity in ex vivo-cultured gut microbial communities. Based on experimentally validated data, we finally discuss the variability amongst and limited accuracy of several prophage prediction tools.

\section{Results}

High throughput sequencing reflects accurate $\mathrm{PtoH}$ ratios in mitomycin $\mathrm{C}$-induced $\mathrm{S}$. Typhimurium cultures

As a model organism for prophage induction, we used a Salmonella enterica serovar Typhimurium LT2 strain (S. $\mathrm{Tm} \mathrm{LT2}{ }^{p 22}$ ). In addition to its original prophages (FELS1, FELS-2, Gifsy-1 and Gifsy-2), S. Tm LT2 ${ }^{p 22}$ carries the prophage p22 [39]. Treatment of $S$. Tm LT2 ${ }^{p 22}$ cultures with mitomycin $\mathrm{C}$ has previously been shown to result in DNA damage, leading to inactivation of prophage repressors $[13,40]$ and subsequent induction of p22 [13, 40]. The induction and replication mode of p22 involves the excision and subsequent circularisation of the phage genome by recombination of homologous sequences present at the attachment sites (attL and $a t t \mathrm{R}$ ) of the prophage genome [41]. Upon excision of the phage genome, recombined attachment sites are generated in the phage (attP) and host (att $\mathrm{B})$ genomes resulting in a circular phage genome and a naïve $S$. Tm $\mathrm{LT}^{p 22}$ strain not carrying the prophage p22 in its genome anymore. In the lytic cycle, concatemeric phage DNA is formed as a result of rolling circle amplification [42], which is packaged into capsids via a mechanism named headful packaging [43]. During this process, DNA is packaged into procapsids initiated from a pac site and proceeding unidirectionally until the carrying capacity of the first procapsid is reached before continuing with packaging DNA into the next procapsid starting wherever the previous one finished [43].

Using this system, we first established quantitative reference values for $\mathrm{PtoH}$ ratios by performing qPCR measurements using induced and non-induced (control) $S$. Tm LT2 $2^{p 22}$ cultures. We designed four qPCR primers (see the 'Methods' section), which, depending on their combination, are indicative of naïve $S$. Tm $\mathrm{LT}^{p 22}$ strains if flanking attB (both primers target bacterial DNA), integrated prophages if flanking att $\mathrm{L}$ (primers target bacterial and viral DNA) and circularised (or concatenated) p22 phage genomes if flanking att $\mathrm{P}$ (both primers target viral DNA) (Fig. 1a/b). In control cultures, we determined a PtoH ratio of $1.07(+/-0.01 \mathrm{sd})$, reflecting a low level of spontaneous phage induction [44]. Upon induction by mitomycin $\mathrm{C}$, the PtoH ratio increased to $14.56(+/-1.18 \mathrm{sd})$. The difference of $\mathrm{PtoH}$ ratios between induced and control cultures $(\Delta \mathrm{PtoH}$ ratio), calculated as the mean $\log _{2}$-fold change $\left(\mathrm{l}_{2} \mathrm{fc}\right)$ of PtoH ratios (Fig. 1c; see also the 'Methods' section: formula iii), was $3.65(+/-0.13 \mathrm{sd})$.

Next, we sought to compare PtoH values based on shotgun-sequenced short insert DNA libraries, from the same samples to the reference values established by qPCR (see the 'Methods' section). To this end, we aligned paired-end $(\mathrm{PE})$ reads from control and induced cultures to a re-sequenced genome (Fig. 2) of $S$. Tm $\mathrm{LT}^{p 22}$ (see Material, Additional file 1: Supplementary Figure 1). The mean PtoH ratio for p22 [1.14 (+/-0.04 sd)], calculated as the fragment coverage of the p22 region divided by the median fragment coverage of 10 universal, single-copy, phylogenetic marker genes $[45,46]$ across replicates, was similar to the $\mathrm{PtoH}$ ratio of $\mathrm{p} 22$ measured by qPCR. In mitomycin C-induced cultures, the $\mathrm{PtoH}$ ratio increased to $14.94(+/-1.73 \mathrm{sd})$ and the $\Delta$ PtoH ratio (Fig. $3 \mathrm{a} / \mathrm{b})$ was $3.71(+/-0.19 \mathrm{sd})$. The PtoH ratios of p22 measured by qPCR and HTS for both control ( $p=0.10$, Welch test; $p=0.19$ paired $t$ test) and induced ( $p=0.77$, Welch test; $p=0.40$ paired $t$ test) $S$. Tm $\mathrm{LT}^{p 22}$ cultures showed no significant difference between the two methods regardless of assuming equal (paired $t$ test) or unequal (Welch test) variances between the groups. The same was true for the $\Delta \mathrm{PtoH}$ ratio between induced and control cultures $(p=0.74$, Welch test; 0.56 paired $t$ test) when measured by qPCR or HTS. The close agreement with the measured qPCR values, therefore, validates our HTS method to determine accurate PtoH ratios.

To determine whether mitomycin $\mathrm{C}$ induces prophages other than $\mathrm{p} 22$, we tested for significant $\Delta \mathrm{PtoH}$ ratios as mean $\mathrm{l}_{2} \mathrm{fc}$ of all genes in $S$. Tm LT2 $2^{p 22}$ using the DESeq2 package [47]. In addition to significantly increased $(p<0.05) \mathrm{l}_{2}$ fcs for each gene in p22 [mean $=3.76$ $(+/-0.13 \mathrm{sd})]$, we identified Fels- 1 as a second induced prophage (Fig. 3a) with a significantly $(p<0.05)$ increased mean $\mathrm{l}_{2} \mathrm{fc}$ of $1.93(+/-0.22 \mathrm{sd})$. This result is in accordance with previous findings demonstrating the inducibility of Fels- 1 by mitomycin C [48]. We also detected some genes within the region of Fels- 2 with significant $\mathrm{l}_{2}$ fc (Fig. 3a). However, no significant $\Delta \mathrm{PtoH}$ ratio was observed for either Gifsy-1 or Gifsy-2, which is in 


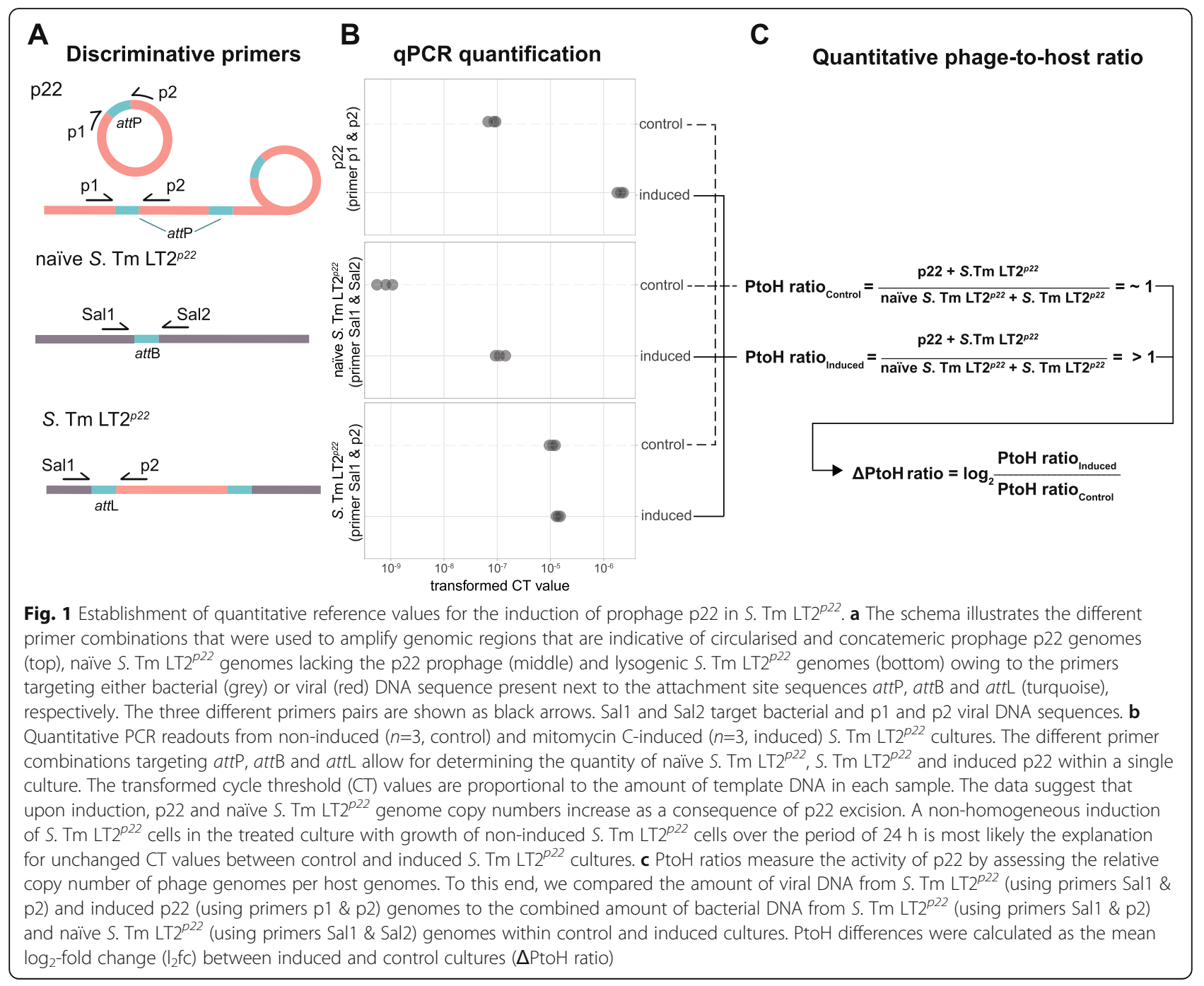

accordance with a lack of virion production of Gifsy-1 and Gifsy-2 after mitomycin C-treatment in Frey et al. (2005). This could be due to mitomycin $C$ having reduced efficiency in Gifsy-1 and Gifsy-2 induction compared to other substances like hydrogen peroxide and/or that the presence of Gifsy-1 may decrease the efficiency of Gifsy-2 induction in S. Tm LT2 strains [49, 50].

\section{Outward-oriented and clipped sequencing reads reveal genomic locations of phages in S. Tm LT2 ${ }^{\text {p22 }}$}

The products of sequencing DNA libraries that contain circularised or concatenated phage genomes, as expected in induced cultures of $S$. Tm LT2 ${ }^{p 22}$, should be composed of PE reads and abnormally distant, outwardoriented PE reads (OPRs) when aligned to the reference genome of the lysogenic host (Fig. 2). This expectation is in accordance with in vitro molecular biology protocols that are used to construct circularised 'mate-pair' DNA libraries of genomic fragments several $\mathrm{kb}$ in size. In this case, the identification of OPRs in reference genome alignments provides information about the original location of the sequenced constructs. Thus, we expected that for induced prophages following this mode of replication, OPRs would accumulate at the attachment sites of prophages $(a t t \mathrm{~L} / \mathrm{R})$ and that once corroborated, identifying OPRs would be a suitable approach to experimentally validate the presence of inducible prophages. In addition, sequencing reads that overlap with the beginning or the end of homologous att core sequences in lytic phages are expected to be only partially aligned (i.e. clipped) at the corresponding att $\mathrm{R}$ and at $\mathrm{L} \mathrm{L}$ sites in the reference genome (Fig. 2). Inspecting these clipped reads should, therefore, allow for identifying the recombining att core sequences of p22 [51], and thus, the exact genomic coordinates of $\mathrm{p} 22$ and other inducible prophages.

In line with these expectations, we observed that mitomycin C-induction led to an increased count of OPRs per cell around the respective $a t t \mathrm{~L}$ and $a t t \mathrm{R}$ sites of $\mathrm{p} 22$, 


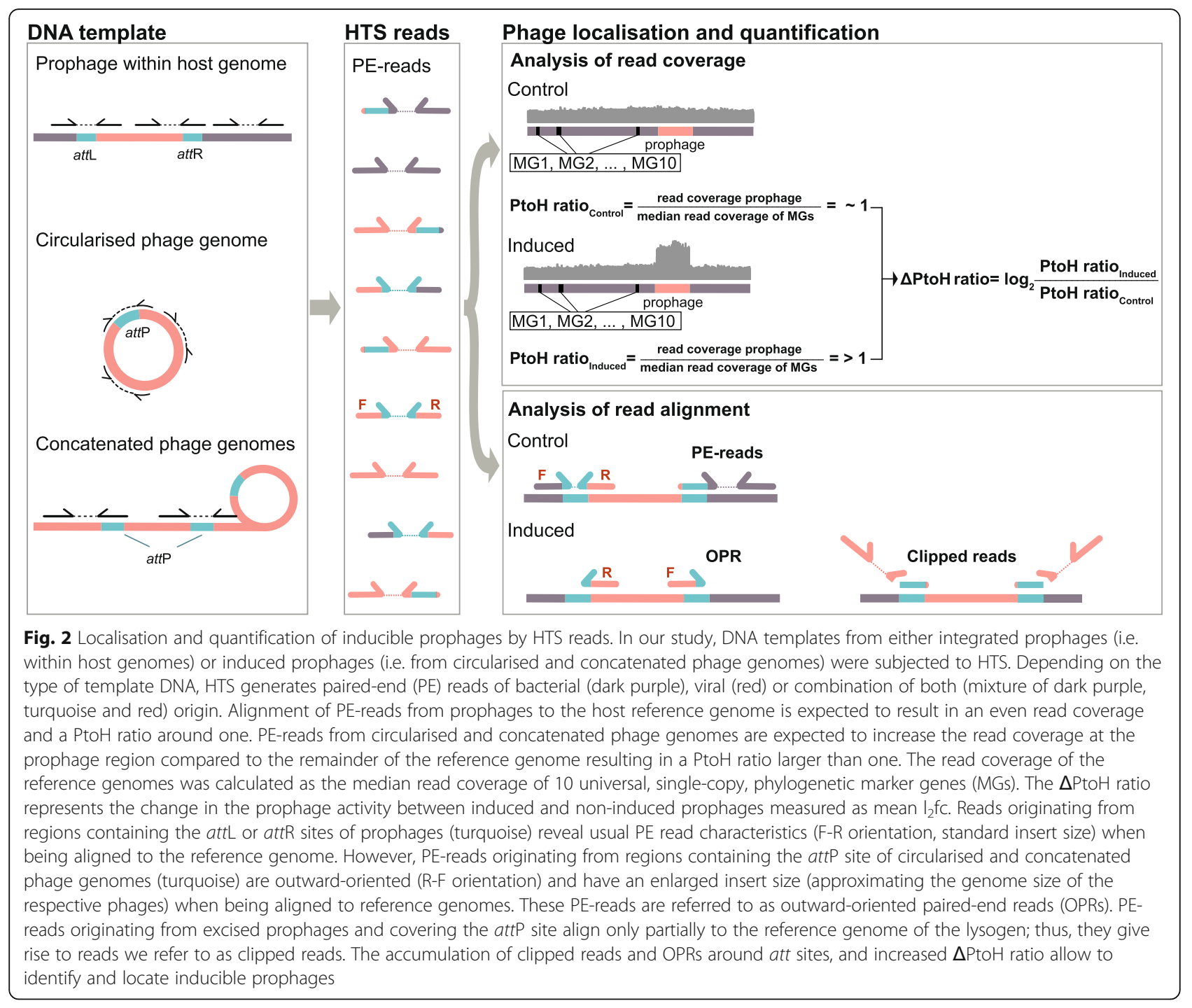

Fels-1 and Fels-2 (Fig. 3b-d). For all three phages, similar counts at the $a t t \mathrm{R}$ and $a t t \mathrm{~L}$ sites provided strong evidence that these OPRs originated from intra-cellular circularised or concatenated phage genomes or mature virions (Additional file 2: Supplementary Table S1). By inspecting clipped reads, we mapped the coordinates of the p22 prophage in $S$. Tm LT2 ${ }^{\mathrm{p} 22}$ to range from $1,213,987$ to $1,255,756$. Furthermore, we found the homologous core sequence of the att site to be in perfect agreement to the one reported initially [51]. When applying the same approach to Fels-1 and Fels-2, we mapped the genomic coordinates of Fels-1 to range from 1,849,458 to 1, 892,188 and verified by clipped read analysis its 8 bp long att core sequence, TCCTTTCA [52]. The coordinates of Fels-2 range from 3,731,215 to 3,764,954 and we identified a $47 \mathrm{bp}$ long att core sequence (Additional file 2: Supplementary Table S3).
Different replication modes are reflected in distinct shapes of read coverage within and adjacent to prophage locations

In addition to analysing OPRs and clipped reads, we observed characteristic patterns within and adjacent to the genomic locations of p22 and Fels-1 in mitomycin C-induced cultures. For example, when comparing control and induced cultures, a stepwise increase in sequenced DNA fragments was observed at the pac site of $\mathrm{p} 22$, followed by a gradual decrease towards the attR site (Fig. 3b). The higher coverage of DNA sequencing reads downstream of the pac site is an expected result of the headful packaging mechanism, which leads to some redundancy of the phage genome within procapsids [43]. Moreover, we observed a significant increase in read coverage of bacterial host genes downstream of the att $\mathrm{R}$ site, resembling a one-sided tent when plotting read 


\section{S. Tm LT2 ${ }^{\text {p22 }}$ from in vitro-induced cultures}

\section{A}

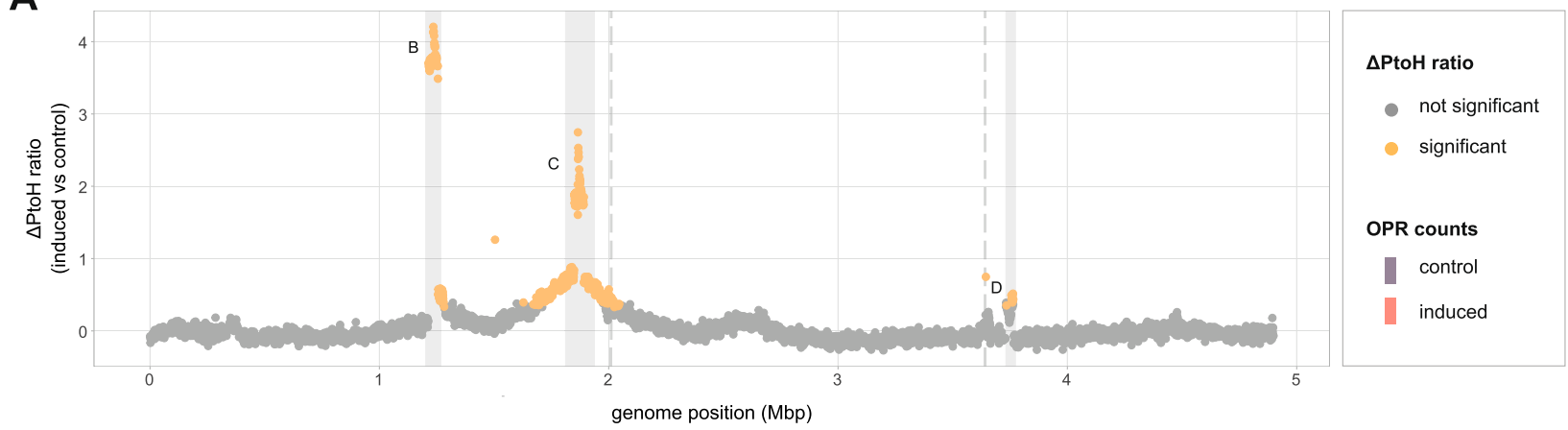

B

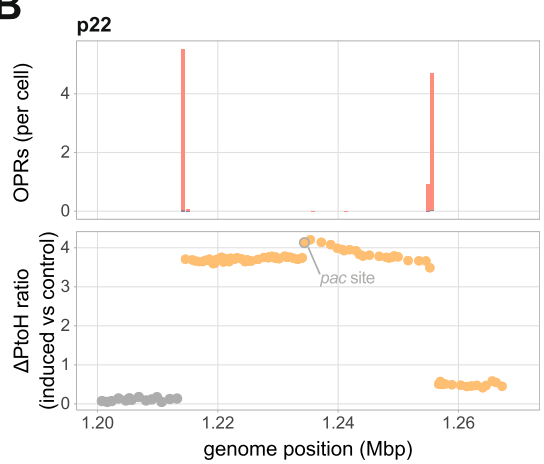

C Fels-1
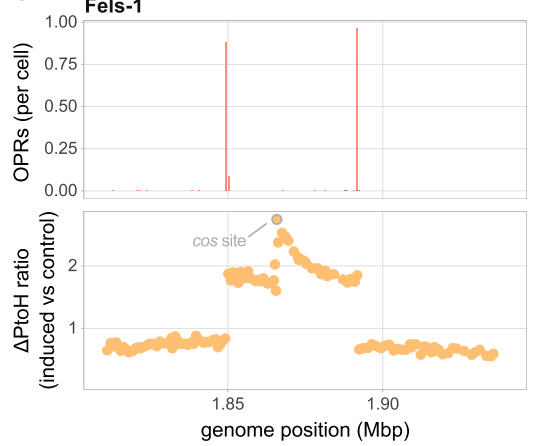

D

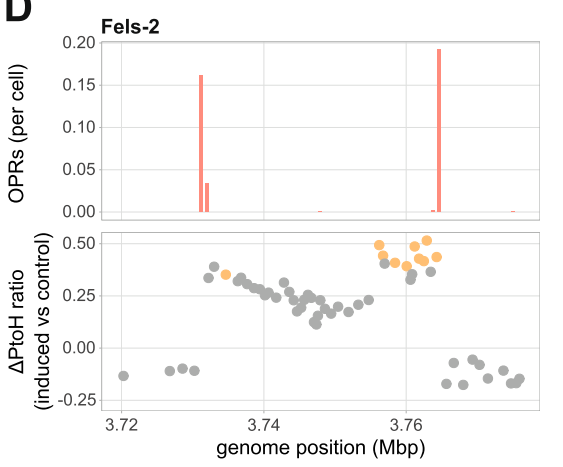

Fig. $3 \Delta$ PtoH ratio and replication modes of induced S. Tm LT2 $2^{p 22}$ prophages determined by high-throughput sequencing. (a) $\Delta$ PtoH ratio of each gene in mitomycin C-induced ( $n=3) S$. Tm LT2 ${ }^{p 22}$ cultures shown as mean $\mathrm{I}_{2} \mathrm{fc}$. The fold change in read coverage for each gene was evaluated using the DESeq2 package. Statistical significance required both a significant change of read coverage between induced and control cultures (Wald test; $p$ value $<0.05$ after Benjamin Hochberg correction) and a $I_{2} f c$ higher than one standard deviation of the mean $I_{2} f c$ of all nonprophage genes. Data points in plots represent all protein-coding S. Tm LT2 ${ }^{p 22}$ genes and are shown in orange if the $\mathrm{I}_{2} f \mathrm{fS}$ were statistically significant (otherwise in grey). The $\mathrm{I}_{2} \mathrm{fcS}$ along the $\mathrm{S}$. Tm $\mathrm{LT} 2^{p 22}$ genome resemble a one-sided and a two-sided tent-like shape around the genomic locations of the phages p22 $(1,213,987-1,255,756)$ and FELS-1 $(1,849,458-1,892,188)$. The grey dashed lines indicate the locations of the prophages Gifsy-2 and Gifsy-1. Detailed views of the genomic locations of the prophages p22 (b), Fels-1 (c) and Fels-2 (d). Mean OPR counts in induced (red) and control (dark purple) cultures are shown in the upper panel. Each bar represents the normalised mean count of OPRs that were aligned within the shown regions. The individual OPR counts per sample were normalised by the sample-specific median insert coverage of 10 single-copy MGs to yield OPR counts per cell before the overall mean OPR counts was calculated. The lower panel shows the patterns of the $\Delta \mathrm{PtoH}$ ratio for each prophage and adjacent host regions. Orange data points with grey borders denote the genes containing the packaging site pac for p22 and cos for Fels-1

coverage against genome position (Fig. 3a). This pattern is likely the result of delayed prophage excision (relative to packaging initiation) resulting in unidirectional headful packaging of integrated viral and adjacent host DNA, which has recently been described as lateral transduction [53]. For Fels-1, we observed a similar stepwise increase within the prophage region, only this time located downstream of a $\cos$ site (Fig. 3c). In contrast to p22, however, host gene DNA fragments were significantly increased both up- and downstream of the att $\mathrm{L}$ and $a t t \mathrm{R}$ sites, respectively, resulting in a characteristic two-sided tent-like shape in the corresponding coverage plot (Fig. 3a). This pattern is likely a result of 'escape replication', during which DNA is bi-directionally amplified in situ prior to excision of Fels-1, as previously described [48]. The 'escape replication' could provide a fitness advantage to the phage by increasing the excision success of Fels-1 or to enhance the change of gene exchange between Fels-1 and Gifsy-2 that is replicated along with Fels-1. Notably, Frye et al. (2005) detected no adjacent host genes in Fels-1 phage particles isolated from mitomycin C-induced $S$. Tm LT2 cultures, which is expected upon excision and $\cos$ site-mediated unit-length packaging of phage procapsids [54]. However, the observed stepwise increase in read coverage downstream of the cos site may rather indicate that immature virions protecting the viral DNA from degradation were produced during escape replication as previously described in excision-defective $\lambda$ prophages [55]. 


\section{OPRs and clipped reads reveal inducible murine gut bacterial prophages}

Having validated our HTS-based methodology to assess the inducibility of prophages, to quantify $\mathrm{PtoH}$ ratios and to map the genomic location of prophages, we sought to apply this approach to investigate previously uncharacterised gastrointestinal prophages. Specifically, we chose to study a set of 12 gut bacterial strains, known as Oligo-MM ${ }^{12}$ [also termed sDMDMm2 (Additional file 2: Supplementary Table S2)], that serve as a representative, defined and experimentally tractable gut microbiota in mice $[56,57]$. Although genome sequences have already been generated, the presence, inducibility and putative replication modes of prophages in these strains have not been characterised to date. In addition, we analysed a human commensal strain, Escherichia coli HS (E. coli HS) [58], one of the predominant facultative anaerobic organisms in the gastrointestinal tract [59].

We aimed to induce putative prophages in cultures of Oligo-MM ${ }^{12}$ strains during the logarithmic growth phase with mitomycin C (Additional file 1: Supplementary Figure S2). To identify inducible prophages, we determined the location of OPRs and tested if the $\Delta \mathrm{PtoH}$ ratio of the genes between OPR had a significant $(p<$ $0.05)$ mean $l_{2} f \mathrm{c}$. We identified two inducible prophages in Akkermansia muciniphila YL44 (YL44), one in Blautia coccoides YL58 (YL58), and another one in E. coli HS (Fig. 4a-c). By analysing clipped read locations, we determined the genomic coordinates for each of the identified prophages (Table 1) and used them as anchor points to identify the homologous core sequence (Additional file 2: Supplementary Table S3,). For YL44, we detected a significant $(p<0.05) \Delta \mathrm{PtoH}$ ratio of $4.41(+/-0.13 \mathrm{sd})$ for the first prophage and a significant $\Delta \mathrm{PtoH}$ ratio of 4.19 $(+/-0.14 \mathrm{sd})$ for the second prophage (Fig. $4 \mathrm{a})$. To verify the production of mature virions, we enriched mitomycin C-induced YL44 cultures for viral particles, sequenced the extracted DNA (see the 'Methods' section), and validated the predicted start and end sites of the inducible prophages by aligning the assembled phage genomes (Additional file 3) to their host genome as well as by clipped read analysis. Although spontaneous induction was observed in control cultures of YL58, we still detected a significant $(p<0.05) \quad \Delta \mathrm{PtoH}$ ratio of 0.61 $(+/-0.05 \mathrm{sd})$ per cell upon mitomycin $\mathrm{C}$ induction (Fig. 4b). For Acutalibacter muris KB18, we observed one and for and Flavonifractor plautii YL31 two additional OPRflanked putative prophage region (Table 1). However, $\Delta \mathrm{PtoH}$ ratios were not statistically significant (Additional file 1: Supplementary Figure S3), since all of these prophages were detected not only in induced but also in control samples. In the case of $E$. coli HS, we identified an inducible prophage with a $\cos$ site (Fig. 4d; see the 'Methods' section; Additional file 2: Supplementary
Table S4). In addition to a significant $\Delta \mathrm{PtoH}$ ratio of $3.51(+/-0.13 \mathrm{sd})$ in the prophage region (Fig. 4d), which was validated by qPCR $(3.53+/-0.16 \mathrm{sd})$, a second region of 229,846 bp length showed a significantly $(p<0.05)$ increased $\Delta \mathrm{PtoH}$ ratio of 0.73 $(+/-0.14 \mathrm{sd})$. However, whereas prophage induction results in an increase in OPRs around the attachment sites of the prophage, no OPR accumulation was observed for the second region (Fig. 4e). Functional annotation of the genes in this region revealed two IS3 family transposases at the start and end of the region.

The read coverage patterns of the newly identified phages in YL44 resembled those we previously observed for $S$. Tm LT2 ${ }^{p 22}$ Fels-1, that is, a two-sided tent suggesting bi-directional escape replication and concomitant production of virions (Fig. 4a). Alignment of sequencing reads from enriched virions to the reference genome of YL44 showed a substantial coverage increase at the locations of both prophages. Together with the accumulation of OPRs at the respective att sites (Fig. 4f), these results suggest that the sequenced DNA originated from mature virions. Notably, read coverages were increased upstream of YL44 phage 1 and downstream of YL44 phage 2, indicating a pac site-mediated lateral transduction potential of both phages (Fig. 4g). These results were corroborated using the software PhageTerm [60] to predict the replication mode of these phages. By contrast, for the induced phage in YL58, we observed a sharp coverage increase (Fig. 4b), similar to the one observed for E. coli HS; however, an accumulation of read start sites, as expected to originate from cos sites with a 5'-cohesive end in linear phage genomes, could not be readily identified.

\section{Gut prophages are inducible in ex vivo-cultured murine gut bacterial community samples}

Having experimentally validated inducible prophages in pure cultures of Oligo- $\mathrm{MM}^{12}$ strains, we investigated the inducibility of prophages from the intestinal community of gnotobiotic Oligo-MM ${ }^{12}$ mice. We evaluated whether the HTS-based approach could detect prophage induction in ex vivo-cultured communities and thus help to evaluate several strains simultaneously. We used mitomycin $\mathrm{C}$ to induce faecal pellets resuspended in media and performed metagenomic sequencing (see the 'Methods' section). By aligning PE reads to the collection of the respective reference genomes, we located OPRs and could verify that the in vitro inducible prophages of YL44 and YL58 produced circular phage genomes when induced in a community setting (Additional file 1: Supplementary Figure S4). In addition to the OPRs detected for the putative prophages in F. plautii YL31 identified 


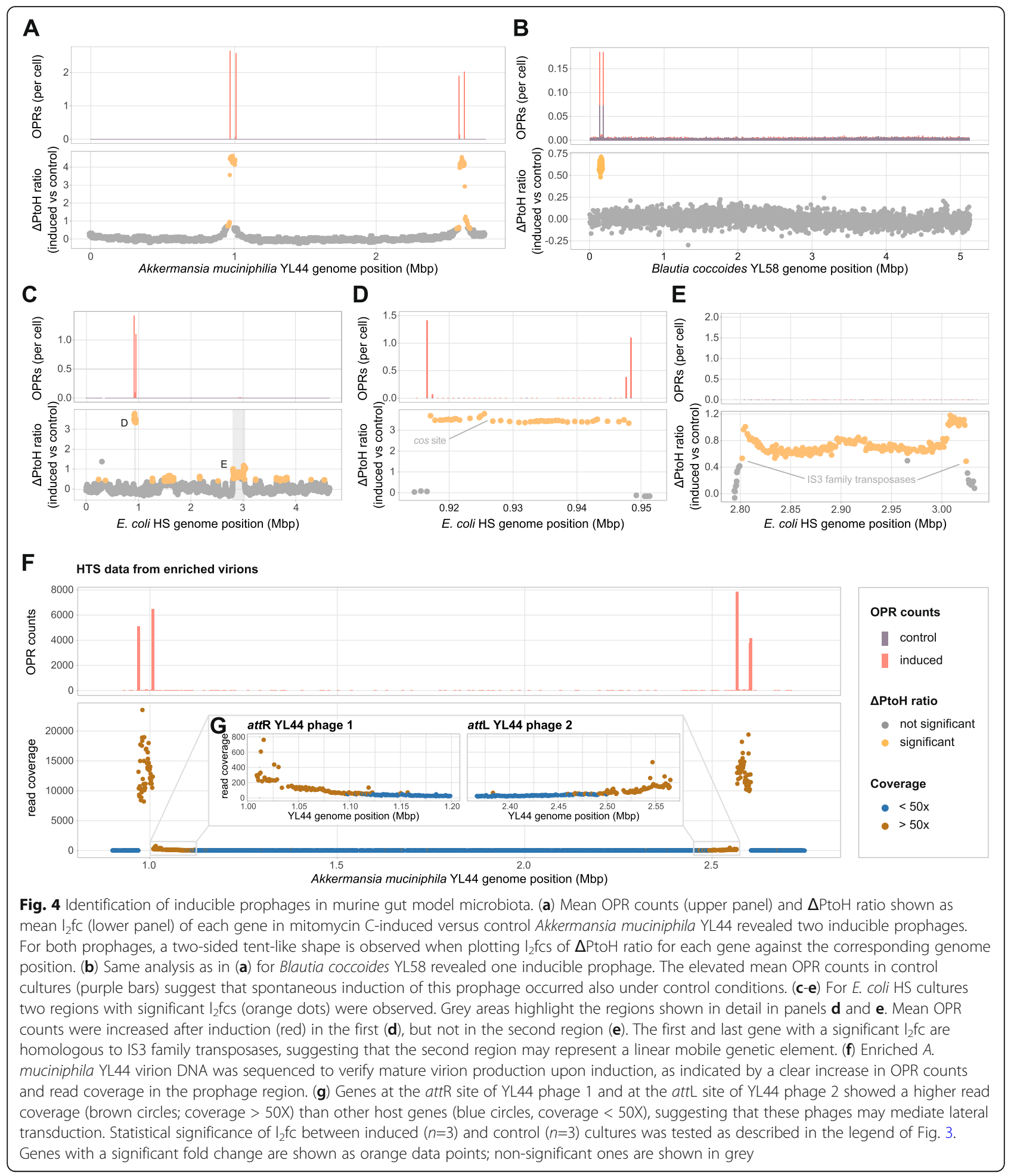

by in vitro induction (Additional file 1: Supplementary Figure S3), we detected a significant $(p<0.05) \Delta \mathrm{PtoH}$ ratio of $2.31(+/-0.16 \mathrm{sd})$ for YL31 phage 1 and 1.95 $(+/-0.11$ sd) for YL31 phage 2 (Fig. 5a-c) through ex vivo-induction. Clipped read inspection specified genomic ranges from 1,009,760 to 1,046,747 (YL31 phage 1) and from 2,984,604 to 3,026,727 (YL31 phage 2). The differential inducibility of YL31 phages between in vitro 
Table 1 Clipped read analysis enables locating coordinates of inducible prophages in gut bacterial genomes

\begin{tabular}{llll}
\hline Strain & Phage & Start site prophage & End site prophage \\
\hline A. muciniphila YL44 & YL44 phage 1 & 969482 & 1008291 \\
& YL44 phage 2 & 2566025 & 2602918 \\
B. coccoides YL58 & YL58 phage 1 & 128601 & 174693 \\
E. coli HS & E. coli HS phage 1 & 916812 & 948289 \\
A. muris KB18 & KB18 phage 1 & 2602856 & 2642096 \\
F. plautii YL31 & YL31 phage 1 & 1009760 & 1046747 \\
& YL31 phage 2 & 2984604 & 3026727
\end{tabular}

Reads originating from circularised or concatenated viral genomes align only partially to the reference genome generating clipped reads. The accumulation of clipped reads at the attL and attR sites allows for identifying the exact start and end coordinates of inducible prophages

\section{Flavonifractor plautii YL31 from ex vivo-induced faecal pellet}

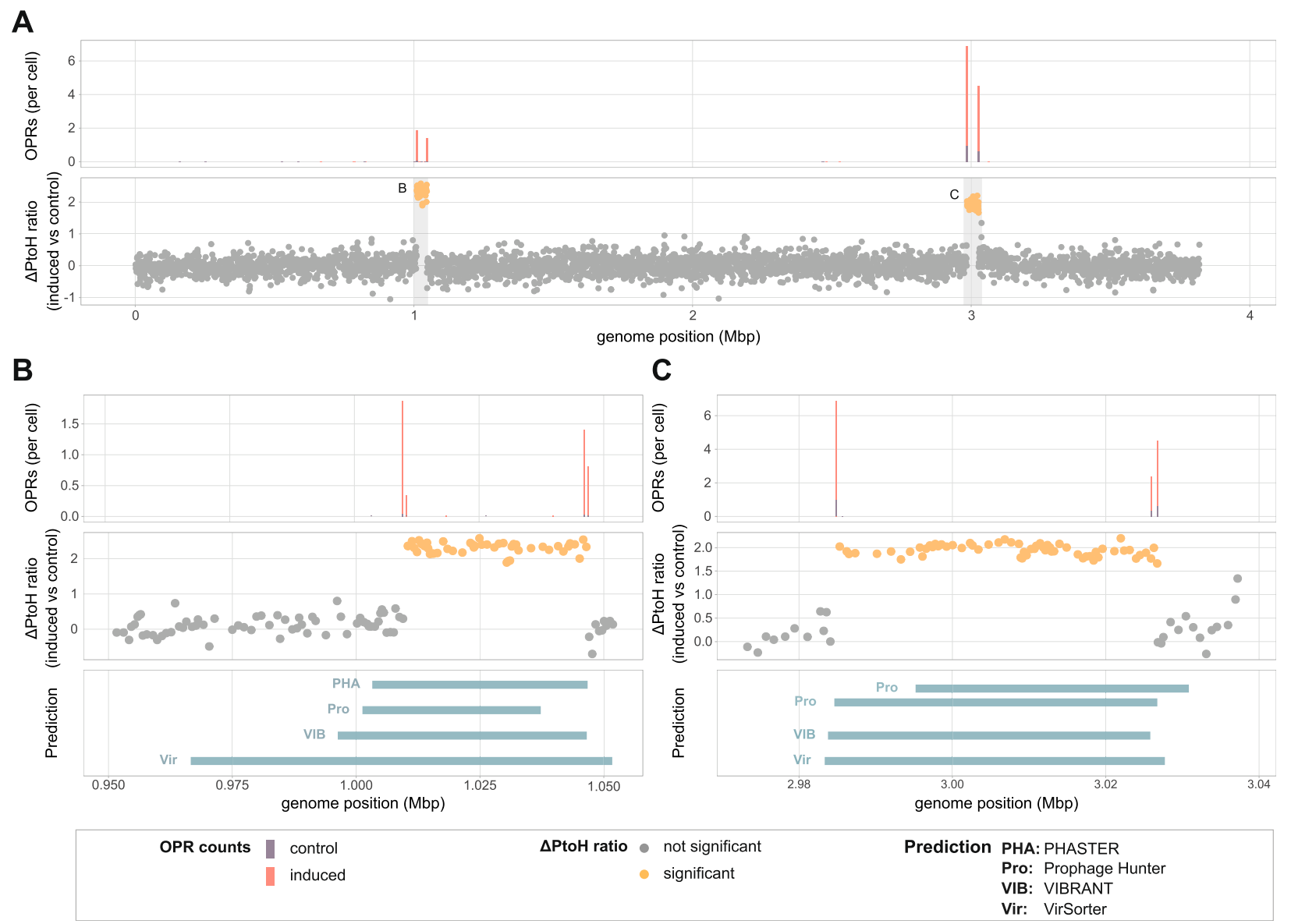

Fig. 5 Detection of inducible prophages in ex vivo-cultured murine faecal samples. (a) Mean OPR counts (upper panel) and $\Delta$ PtoH ratio shown as $\mathrm{I}_{2} \mathrm{fc}$ of genes in the Flavonifractor plautii YL31 genome (lower panel) from mitomycin C-induced versus control cultures of murine, faecal pellets reveal two inducible prophages. The areas highlighted in grey indicate genomic regions of prophage locations that are shown in panels $\mathbf{b}$ and $\mathbf{c}$. (b/c) YL31 phage 1 and YL31 phage 2 show mean OPR counts (upper panels), $\Delta$ PtoH ratio (middle panels) and in silico-predicted regions of prophages in the reference genome of F. plautii YL31 (lower panel) using Prophage Hunter, PHASTER, VIBRANT and VirSorter. Visualisations include prophage predictions of category 1 and 2 for VirSorter, and prophages predicted to be intact or active for PHASTER and Prophage Hunter and predicted prophage of VIBRANT, respectively. None of the prediction tools was able to predict unambiguously start and end coordinates of induced prophages. Statistical significance of differences between induced ( $n=3)$ and control $(n=3)$ cultures of F. plautii YL31 (a-c) was tested as described in the legend of Fig. 3 
and ex vivo conditions may be due to an indirect community response to mitomycin $\mathrm{C}$, or other factors, such as nutrient limitation [61] activating the lytic cycle during ex vivo culturing. We found that our method detected inducible prophages not only in highly abundant strains (Blautia coccoides YL58) but also strains that were present at low relative abundance (A. muciniphila YL44: 1.4\%, F. plautii YL31: 0.9\%). Notably, we detected a high number of OPRs within one region of a strain (Clostridium clostridioforme YL32) with a relative abundance and sequence coverage of as low as $0.55 \%$ and 4.8, respectively (Fig. 6). Despite this evidence for the detection of an additional putative prophage, we did not follow up on this region due to our conservative requirement of a statistically significant $\Delta \mathrm{PtoH}$ ratio in the same region.

\section{In silico predictions of prophage locations are variable and disagree between tools}

Several in silico tools are available to predict prophages in microbial genomes and metagenomic assemblies [3437], which have been used to expand the number of known prophages, especially in bacteria that are challenging to be cultured. Nevertheless, most prediction tools do not account for mutations or biological interactions affecting prophage activity. Therefore, the validity of in silico predictions requires experimental validation to assess the precision in predicting the genomic location and the inducibility of putative prophages. We sought to assess several prediction tools (Additional file 4; Supplementary Table S5-8), for their ability to detect inducible prophages and their accuracy of prophage localisation in reference genomes by comparing their output to our experimentally validated data.
For most predicted prophages ( $>90 \%$ ), we could not verify their inducibility using a sub-lethal concentration of mitomycin C. It is not possible to ascertain whether these prophages were not induced under the specific experimental conditions of our study or whether they cannot be induced at all (e.g. inactive/cryptic prophages). However, for those prophages that were induced in our study in vitro or ex vivo, we found that the predicted locations were in poor agreement with our experimental data as well as inconsistent across the tested tools. For example, Prophage Hunter and VirSorter predicted two alternative prophage locations for YL44 phage 1, which were additionally different between the tools and entirely missed by PHASTER (Table 2). We also sought to obtain a more quantitative measure for tool performance by scoring the precision and sensitivity of the various tools (Table 2) for prophage localisation. As a result, we found that no specific tool outperformed the others. For example, even though Prophage Hunter was the only tool that was able to correctly predict the start and end for a prophage (YL31 phage 2); it also predicted a second possible start and end site for the same prophage (Fig. 5c). VirSorter was the only tool that predicted every prophage, although for two predicted prophages (E. coli phage 1 and p22) it had an equal score with PHASTER (Table 2).

\section{Discussion}

The activity of prophages in host bacteria has previously been studied either by qPCR $[62,63]$ or aligning HTS reads from experimentally enriched virions or faecal samples to reference genomes of lysogens [27, 29, 33]. However, to our knowledge, this is the first time that $\mathrm{PtoH}$ ratios derived from HTS data have been experimentally validated against those obtained by $\mathrm{qPCR}$

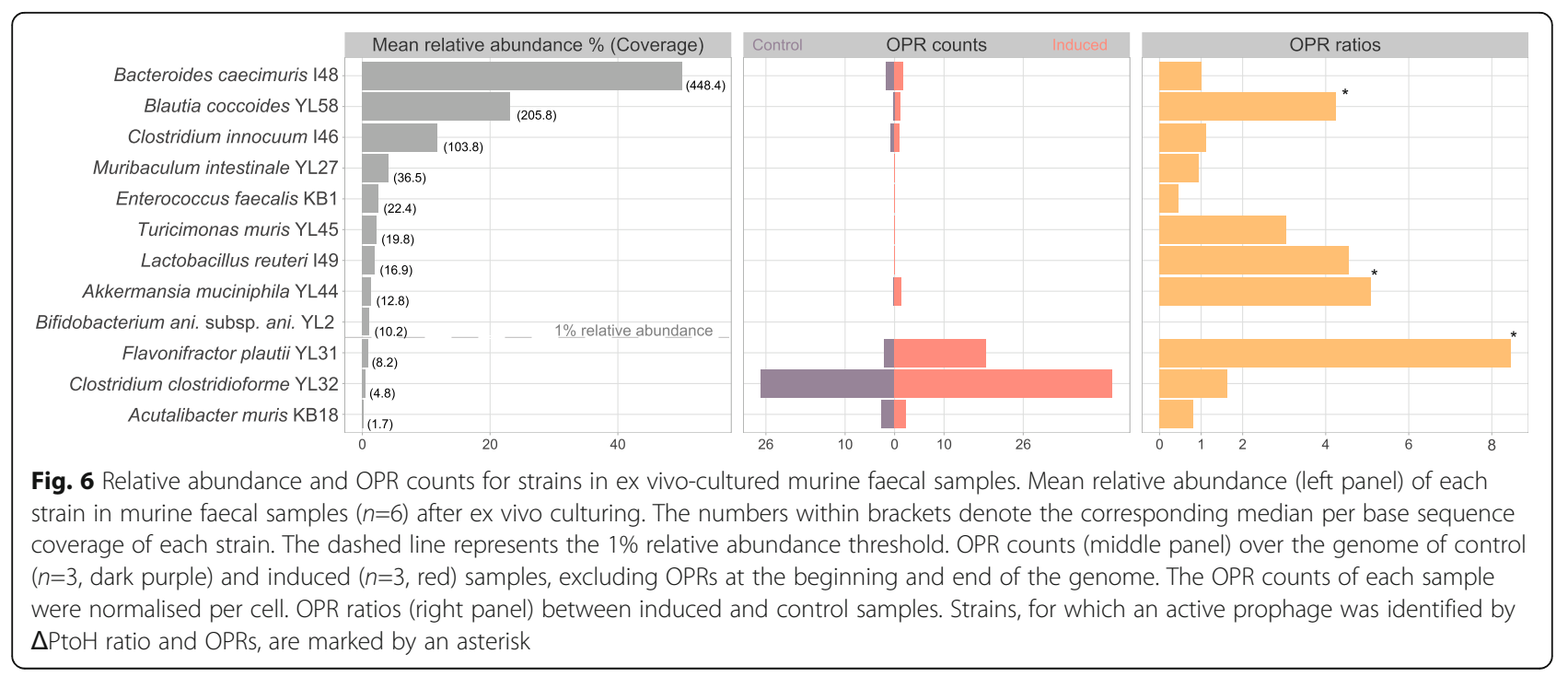


Table 2 Performance evaluation of different tools to predict prophage locations (start/end sites)

\begin{tabular}{|c|c|c|c|c|c|c|c|}
\hline \multirow{3}{*}{$\begin{array}{l}\text { Experimental data } \\
\text { Prophage (range/length—including att } \\
\text { sites) }\end{array}$} & \multicolumn{4}{|l|}{ Prediction } & \multicolumn{3}{|l|}{ Evaluation } \\
\hline & \multirow[t]{2}{*}{ Tool } & \multicolumn{2}{|c|}{ Deviation from } & \multirow[t]{2}{*}{ Length } & \multirow[t]{2}{*}{ Precision } & \multirow[t]{2}{*}{ Recall } & \multirow{2}{*}{$\begin{array}{l}\text { F- } \\
\text { score }\end{array}$} \\
\hline & & Start & End & & & & \\
\hline \multirow[t]{4}{*}{ S. Tm LT2 ${ }^{p 22}$ p22 (1213987-1255756/41770) } & PHASTER & 60 & 1126 & 42836 & 0.97 & 1.00 & 0.99 \\
\hline & Prophage Hunter & -13077 & 8273 & 63120 & 0.66 & 1.00 & 0.80 \\
\hline & VIBRANT & 60 & -334 & 41376 & 1.00 & 0.99 & 1.00 \\
\hline & Virsorter & 10 & 1152 & 42912 & 0.97 & 1.00 & 0.99 \\
\hline \multirow[t]{4}{*}{ S. Tm LT2 ${ }^{p 22}$ Fels-1 (1849458-1892188/42731) } & PHASTER & -1528 & -3284 & 40975 & 0.96 & 0.92 & 0.94 \\
\hline & Prophage Hunter & -4528 & 7083 & 54342 & 0.79 & 1.00 & 0.88 \\
\hline & VIBRANT & 41 & 1216 & 43906 & 0.97 & 1.00 & 0.99 \\
\hline & Virsorter & -20661 & 22091 & 85483 & 0.50 & 1.00 & 0.67 \\
\hline \multirow[t]{4}{*}{ S. Tm LT2 ${ }^{p 22}$ Fels-2 (3731215-3764954/33740) } & PHASTER & 0 & 1410 & 35150 & 0.96 & 1.00 & 0.98 \\
\hline & Prophage Hunter & NA & NA & NA & NA & NA & NA \\
\hline & VIBRANT & 449 & 10659 & 43950 & 0.76 & 0.99 & 0.86 \\
\hline & Virsorter & -115370 & 79199 & 228309 & 0.15 & 1.00 & 0.26 \\
\hline \multirow[t]{6}{*}{ YL44 phage 1 (969482-1008291/38810) } & PHASTER & NA & NA & NA & NA & NA & NA \\
\hline & Prophage Hunter & -9199 & -2567 & 45442 & 0.80 & 0.93 & 0.86 \\
\hline & Prophage Hunter & 23847 & 3354 & 18317 & 0.82 & 0.39 & 0.52 \\
\hline & VIBRANT & 186 & -9968 & 28656 & 1.00 & 0.74 & 0.85 \\
\hline & Virsorter & 1436 & 138 & 37512 & 1.00 & 0.96 & 0.98 \\
\hline & Virsorter & 8087 & 138 & 30861 & 1.00 & 0.79 & 0.88 \\
\hline \multirow[t]{6}{*}{ YL44 phage 2 (2566025-2602918/36894) } & PHASTER & NA & NA & NA & NA & NA & NA \\
\hline & Prophage Hunter & -4041 & -23277 & 17658 & 0.77 & 0.37 & 0.50 \\
\hline & Prophage Hunter & 704 & -2995 & 33195 & 1.00 & 0.90 & 0.95 \\
\hline & Prophage Hunter & 7638 & 10625 & 39881 & 0.73 & 0.79 & 0.76 \\
\hline & VIBRANT & -10070 & -3356 & 43608 & 0.77 & 0.91 & 0.83 \\
\hline & Virsorter & -25233 & 44693 & 106820 & 0.35 & 1.00 & 0.51 \\
\hline \multirow[t]{5}{*}{ YL58 phage 1 (128601-174693/46093) } & PHASTER & NA & NA & NA & NA & NA & NA \\
\hline & Prophage Hunter & -2929 & -27047 & 21975 & 0.87 & 0.41 & 0.56 \\
\hline & Prophage Hunter & 139 & -49 & 45905 & 1.00 & 1.00 & 1.00 \\
\hline & VIBRANT & 153 & 3341 & 49281 & 0.93 & 1.00 & 0.96 \\
\hline & Virsorter & -1242 & 33898 & 81233 & 0.57 & 1.00 & 0.72 \\
\hline \multirow[t]{4}{*}{ E. coli HS phage 1 (916812-948289/31478) } & PHASTER & 78 & 3104 & 34504 & 0.91 & 1.00 & 0.95 \\
\hline & Prophage Hunter & NA & NA & NA & NA & NA & NA \\
\hline & VIBRANT & 461 & 4880 & 35897 & 0.86 & 0.99 & 0.92 \\
\hline & Virsorter & 2787 & -41 & 28650 & 1.00 & 0.91 & 0.95 \\
\hline \multirow[t]{4}{*}{ YL31 phage 1 (1009760-1046747/36988) } & PHASTER & -6510 & -17 & 43481 & 0.85 & 1.00 & 0.92 \\
\hline & Prophage Hunter & -8493 & -9478 & 36003 & 0.76 & 0.74 & 0.75 \\
\hline & VIBRANT & -13474 & -185 & 50277 & 0.73 & 0.99 & 0.84 \\
\hline & Virsorter & -43160 & 4955 & 85103 & 0.43 & 1.00 & 0.61 \\
\hline \multirow[t]{5}{*}{ YL31 phage 2 (2984604-3026727/42124) } & PHASTER & NA & NA & NA & NA & NA & NA \\
\hline & Prophage Hunter & 0 & 0 & 42124 & 1.00 & 1.00 & 1.00 \\
\hline & Prophage Hunter & 10596 & 4114 & 35642 & 0.88 & 0.75 & 0.81 \\
\hline & VIBRANT & -828 & -914 & 42038 & 0.98 & 0.98 & 0.98 \\
\hline & Virsorter & -1257 & 983 & 44364 & 0.95 & 1.00 & 0.97 \\
\hline
\end{tabular}


Table 2 Performance evaluation of different tools to predict prophage locations (start/end sites) (Continued)

\begin{tabular}{|c|c|c|c|c|c|c|c|}
\hline \multirow{3}{*}{$\begin{array}{l}\text { Experimental data } \\
\text { Prophage (range/length—including att } \\
\text { sites) }\end{array}$} & \multicolumn{4}{|l|}{ Prediction } & \multicolumn{3}{|c|}{ Evaluation } \\
\hline & \multirow[t]{2}{*}{ Tool } & \multicolumn{2}{|c|}{ Deviation from } & \multirow[t]{2}{*}{ Length } & \multirow[t]{2}{*}{ Precision } & \multirow[t]{2}{*}{ Recall } & \multirow{2}{*}{$\begin{array}{l}\text { F- } \\
\text { score }\end{array}$} \\
\hline & & Start & End & & & & \\
\hline \multirow[t]{4}{*}{ KB18 phage 1 (2602856-2642096/39241) } & PHASTER & -5132 & 7663 & 52036 & 0.75 & 1.00 & 0.86 \\
\hline & Prophage Hunter & 3156 & 15738 & 51823 & 0.70 & 0.92 & 0.79 \\
\hline & VIBRANT & NA & NA & NA & NA & NA & NA \\
\hline & Virsorter & -45270 & 5505 & 90016 & 0.44 & 1.00 & 0.61 \\
\hline
\end{tabular}

The accuracy of prediction tools to locate prophages was evaluated by comparing the predicted start and end coordinates to the corresponding data obtained by experimental validation. The assessment includes the recall, precision and F-score of correctly predicting prophage genomic coordinates. The first column reports the range and length of each prophage based on experimental data as determined by analysis of clipped reads. Columns two to five summarise the results of the different prediction tools. The deviations from the validated start and end positions are shown with negative and positive numbers if the predicted site was upstream or downstream of the true start or end site, respectively. For each prophage, the tool with the best F-score is highlighted in bold. In the case of a tie, both tools with the highest F-score are highlighted. Prophages that were not categorised as 'active' (Prophage Hunter), 'complete' (PHASTER), 'category 1' or 'category 2' (VirSorter), or not predicted at all, are reported as NA

measurements. Although associated with higher costs, one major advantage of the HTS-based approach is that the relative activity of induced prophages can be quantified without prior knowledge of the location of att sites in the host genome. As shown here for several bacterial strains from a defined gut microbiota collection, the location of inducible prophages that form circularised intermediate products during replication can be approximated by identifying OPRs and precisely determined by inspecting clipped read alignments. However, our results also show that verification of the presence of viral hallmark genes is needed to distinguish prophages from other circularising mobile genetic elements that may also be transferred as dsDNA [64, 65].

We found that both the availability of appropriate reference genomes (Additional file 1; Supplementary Figure S1) and experimental validation of prophage regions can be beneficial for an accurate quantification of PtoH ratios. The demonstrated applicability of the OPR-based approach to ex vivo-induced faecal samples and the subsequent localisation of a prophage within a strain with less than $5 \mathrm{x}$ sequence coverage highlights the potential of the presented approach to study inducible prophages of microbiota members that are present at low relative abundances, such as F. plautii YL31 in the Oligo-MM ${ }^{12}$ model [56]. However, low sequence coverage may not allow for the detection of specific replication patterns, and it should be noted that PtoH ratios measured in cultures of individual strains or communities only represent average values for a sampled population. To study the burst size of prophages or to resolve spatio-temporal heterogeneities in prophage activity within microbial populations will require determining these ratios at single-cell resolution.

Our results on comparing the performance of a selection of prophage prediction tools revealed that experimental validation is currently unmatched in terms of accuracy for determining the location of prophages, even of known ones, within bacterial host genomes. Furthermore, in our analyses, only a fraction of the predicted prophages were inducible using a single stimulus. It may thus be possible that prediction tools overestimate the number of inducible prophages by detecting and mislabelling cryptic prophages, which may constitute a sizable fraction of a species' accessory genome [66]. However, the presented methods are restricted to locate complete, host-integrated, lysogenic prophages with circularised and/or concatenated genomes produced upon their induction and excision. Thus, other predicted prophages, despite being inducible, may not have responded to the experimental conditions tested here. In future studies, the use of an extended spectrum of induction stimuli may help to improve our knowledge about inducible, circularising prophages in a variety of bacteria, and help to populate virus-centric databases and to improve the accuracy of bioinformatic prediction tools.

\section{Conclusions}

To improve our understanding of phage-microbe interactions and their relevance in mammalian health and disease, it is critical to overcome current challenges in locating inducible prophages and in quantifying their activity in gut microbial communities. The present work demonstrates the suitability of experimental induction and subsequent analysis of HTS data as a powerful approach to tackle these challenges using gut microbial strains cultured either individually or as communities starting from mouse faecal samples. Experimental validation of predicted prophages remains an important task for the annotation of bacterial genomes, given that predictions from bioinformatics tools alone may be incongruent and insufficiently accurate. 


\section{Methods}

Induction and extraction of Salmonella enterica serovar Typhimurium $\mathrm{LT}^{\text {p22 }}$

Salmonella enterica serovar Typhimurium LT2 carrying a lysogenic p22 phage $\left(S\right.$. Tm LT2 $\left.{ }^{p 22}\right)$ was cultured overnight in lysogeny broth (LB; $10 \mathrm{~g} \mathrm{~L}^{-1}$ tryptone (Oxoid), 5 $\mathrm{g} \mathrm{L}^{-1}$ yeast extract (Oxoid), $5 \mathrm{~g} \mathrm{~L}^{-1} \mathrm{NaCl}$ (Thermo Fisher)) supplemented with $1.23 \mathrm{~g} \mathrm{~L}^{-1} \mathrm{MgSO}_{4} \cdot 7 \mathrm{H}_{2} \mathrm{O}$ (Sigma-Fluka) before it was diluted 1:20, subcultured for $4 \mathrm{~h}$ and further diluted to reach a final concentration of $10^{5}$ bacteria $\mathrm{mL}^{-1}$. We separated the cultures into control and induced cultures. The induced cultures were supplemented with $1 \mu \mathrm{g} \mathrm{mL} \mathrm{m}^{-1}$ mitomycin C (Sigma-Aldrich), and all cultures were aerobically incubated for 24 $\mathrm{h}$ at $37{ }^{\circ} \mathrm{C}$ and shaken at $180 \mathrm{~min}^{-1}$ (Kühner Shaker). We used $100 \mu \mathrm{L}$ of each culture for DNA extraction using the AllPrep DNA/RNA Kit (Qiagen) with the following modification in the disruption step: the samples were mixed with $0.6 \mathrm{~mL}$ of RLT buffer complemented with $10 \mu \mathrm{L}$ 2-beta-mercaptoethanol (Sigma-Aldrich), added to $2 \mathrm{~mL}$ tubes pre-filled with $100 \mu \mathrm{m}$ Zirconium beads (OPS Diagnostics LLC) and disrupted twice at 30 $\mathrm{Hz}$ for $3 \mathrm{~min}$ with $5 \mathrm{~min}$ incubation between each disruption step using the mixer mill Retsch MM400. Samples were centrifuged at full speed for $1 \mathrm{~min}$ to pellet the cell debris and then transferred to DNA-binding columns. We eluted the DNA in $60 \mu \mathrm{L}$ elution buffer (EB) and used it as templates for qPCRs and high-throughput sequencing. Library preparation using the TruSeq Nano DNA Library Kit (Illumina) and sequencing was carried out by the Functional Genomics Centre Zurich using the NovaSeq 6000 platform and $2 \times 150$ bp PE-reads with a target insert size of $500 \mathrm{bp}$. For each sample, the output was on average $6.9 \mathrm{M}$ reads.

\section{In vitro induction of murine intestinal model bacterial strains}

Oligo-MM ${ }^{12}$ strains [56, 57] were individually cultured for 12-24 $\mathrm{h}$ in supplemented brain heart infusion medium (BHIS) (37 $\mathrm{g} \mathrm{L}^{-1}$ brain heart infusion broth (Thermo Fisher), $2.5 \mathrm{mg} \mathrm{L}^{-1}$ hemin (Sigma-Aldrich), $1.23 \mathrm{~g} \mathrm{~L}^{-1} \mathrm{MgSO}_{4} \cdot 7 \mathrm{H}_{2} \mathrm{O}$ (Sigma-Fluka), $2 \mathrm{~g} \mathrm{~L}^{-1}$ $\mathrm{NaHCO}_{3}$ (Sigma-Aldrich), $500 \mathrm{mg} \mathrm{L}{ }^{-1}$ L-cysteine (Sigma-Aldrich), $500 \mu \mathrm{g} \quad \mathrm{L}^{-1}$ menadione (Sigma-Aldrich)). The two exceptions were Akkermansia muciniphila YL44 for which the BHIS was additionally supplemented with $25 \mathrm{mg} \mathrm{L}^{-1}$ mucin (Sigma-Aldrich) and Lactobacillus reuteri I49, which was cultured in $51 \mathrm{~g}$ $\mathrm{L}^{-1}$ MRS broth supplemented with $1 \mathrm{~mL} \mathrm{~L}^{-1}$ Tween 80 and $1.23 \mathrm{~g} \mathrm{~L}^{-1} \mathrm{MgSO}_{4} \cdot 7 \mathrm{H}_{2} \mathrm{O}$ (Sigma-Fluka). The cultures were diluted $10^{-1}$ to the final volume of $6 \mathrm{~mL}$ and sub-cultured for 1 to $15 \mathrm{~h}$ to reach the logarithmic growth phase before each culture was divided equally into control and induced cultures. The induced cultures were supplemented with $1 \mu \mathrm{g} \mathrm{mL} \mathrm{m}^{-1}$ mitomycin $\mathrm{C}$ (Sigma-Aldrich) and both cultures were incubated at 37 ${ }^{\circ} \mathrm{C}$ for 1-3 days. Escherichia coli HS was cultured as described for $S$. Tm LT2 ${ }^{p 22}$ with the exception of using BHIS instead of LB. All the handling and culturing of the Oligo-MM $\mathrm{M}^{12}$ and E. coli $\mathrm{HS}$ strains were carried out in an anoxic atmosphere $\left(7 \% \mathrm{H}_{2}, 10 \% \mathrm{CO}_{2}\right.$, rest $\left.\mathrm{N}_{2}\right)$. Afterwards, the cultures were transferred to Amicon tubes (MWCO 10,000), centrifuged at $3400 \times g$ for 30 min and washed with SM buffer $\left(5.8 \mathrm{~g} \mathrm{~L}^{-1} \mathrm{NaCl}, 2 \mathrm{~g}\right.$ $\mathrm{L}^{-1} \mathrm{MgSO}_{4} \cdot 7 \mathrm{H}_{2} \mathrm{O}$ and $50 \mathrm{mM} \mathrm{L}^{-1}$ Tris- $\mathrm{HCl} \mathrm{pH} \mathrm{7.4)}$ before finally being concentrated to $200 \mu \mathrm{L}$. For additional cell lysis, the concentrate was mixed with 174 $\mu \mathrm{L}$ TE-buffer (30 $\mathrm{mM}$ Tris- $\mathrm{HCl}$ and $1 \mathrm{mM}$ EDTA) supplemented with $30 \mathrm{mg} \mathrm{mL} \mathrm{m}^{-1}$ Lysozyme (Sigma-Aldrich), $1.6 \mathrm{U} \mathrm{mL}^{-1}$ Proteinase $\mathrm{K}$ (NEW ENGLAND BioLabs) and $10 \mathrm{U} \mathrm{mL}^{-1}$ Mutanolysin (Sigma-Aldrich) and incubated at $37{ }^{\circ} \mathrm{C}$ for $30 \mathrm{~min}$. The DNA was finally extracted following the same protocol used for $S$. Tm LT2 ${ }^{p 22}$ with the adjustment that the volume of RLT-buffer was increased to $0.75 \mathrm{~mL}$. Library preparation and sequencing was carried out by Novogene using the NEBNext DNA Library Prep Kit with a target insert size of $350 \mathrm{bp}$ and using the NovaSeq 6000 platform with $2 \times 150$ bp PE-reads, respectively. For each sample, the output was on average $10.2 \mathrm{M}$ reads.

\section{Ex vivo induction of murine gut bacterial community samples}

We collected the fresh faecal pellets from three cohoused mice and separated them into halves for control and induced cultures. We homogenised each half in $0.5 \mathrm{~mL}$ anoxic BHIS medium containing one 3 mm metal bead at $10 \mathrm{~Hz}$ for 30 using the mixer mill Retsch MM400. The homogenised cultures were transferred into Hungate tubes containing $5 \mathrm{~mL}$ prewarmed anoxic BHIS medium. The induced cultures were treated with $1 \mu \mathrm{g} \mathrm{mL} \mathrm{m}^{-1}$ mitomycin $\mathrm{C}$ and anaerobically cultured with the control samples at $37{ }^{\circ} \mathrm{C}$, shaken at $180 \mathrm{~min}^{-1}$ (Kühner Shaker) for $6 \mathrm{~h}$. From each culture, $200 \mu \mathrm{L}$ were pretreated with $174 \mu \mathrm{L}$ TE-buffer (30 mM Tris- $\mathrm{HCl}$ and $1 \mathrm{mM}$ EDTA) supplemented with $30 \mathrm{mg} \mathrm{mL}^{-1}$ Lysozyme (Sigma-Aldrich), $1.6 \mathrm{U} \mathrm{mL}^{-1}$ Proteinase $\mathrm{K}$ (NEW ENGLAND BioLabs) and $10 \mathrm{U} \mathrm{mL}^{-1}$ Mutanolysin (Sigma-Aldrich) and incubated at $37{ }^{\circ} \mathrm{C}$ for $30 \mathrm{~min}$ before the DNA was extracted as described above for $S$. Tm LT2 ${ }^{p 22}$. The library preparation using TruSeq Nano DNA Library Kit (Illumina) and the sequencing was carried out by the Functional Genomics Centre Zurich using the NovaSeq 6000 platform and $2 \times 150$ bp PE-reads with a target insert size of $500 \mathrm{bp}$. For each sample, the output was on average $28.2 \mathrm{M}$ reads. 


\section{Akkermansia muciniphila YL44 virion enrichment and sequencing}

For the extraction of free Akkermansia muciniphila YL44 virions, $1 \mathrm{~L}$ of axonic BHIS medium supplemented with $25 \mathrm{mg} \mathrm{L}^{-1}$ mucin (Sigma-Aldrich) was inoculated with $10^{6}$ cells $\mathrm{mL}^{-1}$ of Akkermansia muciniphila YL44 and sub-cultured for $11 \mathrm{~h}$ at $37{ }^{\circ} \mathrm{C}$ before induction with mitomycin $\mathrm{C}\left(1 \mu \mathrm{g} \mathrm{mL}^{-1}\right)$. After $48 \mathrm{~h}$ of induction at 37 ${ }^{\circ} \mathrm{C}$, the virions were separated from the bacteria by two repeated centrifugation steps at $7000 \times g$ for $30 \mathrm{~min}$ using a Sorvall RC-6 centrifuge (Thermo SCIENTIFIC) and an additional filtration step using a $0.22-\mu \mathrm{m}$ bottle filter (Filter Top 500 (TPP)). Between the centrifugation steps, the supernatant was transferred into a new sterile bucket without disturbing the pellet. To precipitate the free virions, $1 \mathrm{~mL}$ of $10 \mathrm{~g} \mathrm{~L}^{-1} \mathrm{FeCl}_{3} \cdot 6 \mathrm{H}_{2} \mathrm{O}$ was added to the supernatant, mixed and incubated at room temperature for $1 \mathrm{~h}$ before centrifugation at $27,000 \times g$ for $1 \mathrm{~h}$ using a Sorvall LYNX 6000 centrifuge (Thermo SCIENTIFIC). Afterwards, the supernatant was removed without disturbing the pellet, and the pellet subsequently resuspended in $3 \mathrm{~mL}$ ascorbic-EDTA buffer $\left(0.4 \mathrm{M} \mathrm{Mg}_{2}\right.$ EDTA and 0.8 M ascorbic acid, pH 6-7) before concentrated using Amicon tubes (MWCO 10.000). The concentrate was washed three times using $15 \mathrm{~mL}$ SM buffer before it was finally concentrated to $200 \mu \mathrm{L}$. The concentrate was treated with $50 \mathrm{U} \mathrm{mL}^{-1}$ DNase I (Thermo SCIENTIFIC) and RNase H (NEW ENGLAND BioLabs) at $37{ }^{\circ} \mathrm{C}$ for $2 \mathrm{~h}$ to remove free DNA and RNA before the reaction was interrupted with $45 \mu \mathrm{L} 0.5 \mathrm{M}$ EDTA. Before the extraction (using the standard extraction protocol), the concentrate was incubated with $3.8 \mathrm{U}$ Proteinase $\mathrm{K}$ at $50{ }^{\circ} \mathrm{C}$ for $30 \mathrm{~min}$ and centrifugation at 17 , $000 \times g$ for $3 \mathrm{~min}$ to remove cell debris. The DNA was eluted in $100 \mu \mathrm{L}$ EB buffer and concentrated to $15 \mu \mathrm{L}$ using the genomic DNA clean \& concentrator kit (Zymo Research). The viral DNA was sequenced at Novogene using the NovaSeq 6000 platform and $2 \times 150$ bp PEreads with a target insert size of $350 \mathrm{bp}$. For the virion sample, the output was $12.6 \mathrm{M}$ reads.

\section{NanoPore re-sequencing and assembling of Salmonella enterica serovar Typhimurium $\mathrm{LT}^{\text {p22 }}$}

$S$. Tm LT $2^{p 22}$ was cultured as described above with the modification that a $30 \mathrm{~mL}$ subculture was used for the extraction of high molecular weight DNA using the NucleoBond AXG Columns (Macherey-Nagel) and NucleoBond Buffer Set III (Macherey-Nagel) following the supplier's protocol. As recommended, the G3 buffer was supplemented with $100 \mathrm{mg} \mathrm{mL}^{-1}$ Lysozyme (SigmaAldrich), and the final DNA pellet was additionally washed with $70 \%$ ethanol before it was redissolved for 1 $\mathrm{h}$ at $55{ }^{\circ} \mathrm{C}$ in $150 \mathrm{~mL} \mathrm{~EB}$ buffer (Qiagen). The DNA quality and quantity were checked by a NanoDrop photo spectrometer (260/280: 1.89 and 260/230: 2.06) and a Qubit $^{\mathrm{TM}} 4$ Fluorometer (Invitrogen), respectively, before 400 ng of DNA was used as input for library preparation (SQK-RAD004). Sequencing was performed on a MinION R9 flow cell (Oxford Nanopore Technologies) for $24 \mathrm{~h}$, producing approximately $11.5 \mathrm{Gbp}$ read data, with a median read length of just over $2800 \mathrm{bp}$. Reads were basecalled with Guppy-CPU (v.3.2.4+d9ed22f; Oxford Nanopore Technologies) with default parameters. Adaptors were trimmed with Porechop (v0.2.4) [67] and then filtered to keep only the highest quality $500 \mathrm{Mbp}$ with Filtlong (v.0.2.0) [68]. Assembly was performed with Flye (v.2.4.1) [69] aiming for a genome size of $5 \mathrm{Mbp}$, resulting in two contigs of size $4.95 \mathrm{Mbp}$ and $95 \mathrm{kbp}$. The assembly was polished with five cycles of Pilon (v.1.23) [70], using the Illumina reads, aligned with BWA mem (v.0.7.17-r1188) [71], which resulted in final contig sizes of $4.9 \mathrm{Mbp}$ and $94 \mathrm{kbp}$.

\section{Quality control of high-throughput sequencing data}

Raw sequencing data were quality controlled using the command bbduk.sh of BBMap (v.38.71) [72]. First, we removed adapters from the reads, followed by the removal of reads that mapped to quality control sequences (PhiX genome). We discarded low quality reads by applying the parameters trimq $=14, \operatorname{maq}=20, \max n s=1$ and minlength $=45$.

\section{Gene abundance calculation}

The quality-controlled forward and reverse reads were mapped against the respective host genome using the bwa mem (v.07.17-r1188) algorithm [71] in PE mode and using the $-a$ flag. Alignments were filtered to be at least 45 bases in length, with an identity of $\geq 97 \%$ to the reference genome and covering $\geq 80 \%$ of the query sequence. The resulting alignment file was used as input for featureCounts (v.2.0.0) [73] allowing multioverlapping reads and multi-mapping reads $(-O-M$ --fraction) to retrieve gene counts. Inserts were counted instead of reads $(-p)$ and incomplete inserts with only one aligned read were discarded $(-B)$. The statistical analysis of differential insert counts per gene in control and induced samples was done in RStudio (v1.2.5042) with $\mathrm{R}$ (v.3.6.3) using the command DEseq from the DESeq2 (v.1.22.2) package, applying mean as fitType for dispersion fitting. As output, DESeq 2 provided $\log _{2}$-fold change $\left(\mathrm{l}_{2} \mathrm{fc}\right)$ values and $p$ values based on Wald tests, adjusted by the Benjamin-Hochberg procedure. For statistically significant changes between induced and control cultures, we required an adjusted $p$ value $<0.05$ and an $l_{2} f c$ more than one standard deviation away from the mean $\mathrm{l}_{2} \mathrm{fc}$ of all non-prophage genes. 
Identification and localisation of prophages in lysogenic host genomes by OPRs and clipped reads

Quality controlled forward and reverse reads were mapped individually against the respective host genome using bwa mem (version 0.7.17-r1188) with the -a flag.. Alignments were filtered to be at least 45 bases in length, with an identity of $\geq 97 \%$ to the reference genome and covering $\geq 80 \%$ of the query sequence. OPR detection was performed with the mVIRs package (version 1.0, https://github.com/SushiLab/mVIRs). To detect OPRs, reads were grouped to inserts by name and high and low boundaries for insert sizes of properly paired reads were estimated using the mean insert sizes and \pm seven standard deviations of uniquely mapped inwardoriented paired-end reads (IPRs). OPRs were detected as follows: For each insert, first, find the best scoring alignment pairs within $3 \%$ of the best alignment score. Second, report the insert as OPR if there is no IPR with reasonable insert size within the $3 \%$ cutoff and if the OPR is the best scoring alignment. To evaluate the mean OPR count per cell, we split the genome into bins with the size of the median gene length of the respective bacterial genome. For each OPR, we identified the two bins into which the forward and the reverse reads fell and calculated the midpoint of each bin used as the input for undirected graph building using the command graph from_data_frame from the package igraph (v.1.2.4.2). We excluded OPR pairs with an insert size smaller than $1000 \mathrm{bp}$ and larger than host genome length minus the genome length of smallest circular dsDNA bacteriophage on NCBI [74] for the graph building. We extracted the edges, which represent the OPRs, using the command get.edgelist and counted the edges with the same coordinates. The sum was normalised by the median coverage of 10 universal, single-copy, phylogenetic marker genes (MGs) and the bin length to evaluate the number of OPRs per cell. As the last step, we calculated the mean OPR count for each bin from the triplicates. OPRs falling into the surrounding regions of the left and right attachment site $+/$ - three times the insert size was summed up to verify that the number of OPRs at attR and $a t t \mathrm{~L}$ sites were the same.

For the precise determination of the start and end position of a prophage, the quality-controlled reads were individually mapped as described above. We sorted, indexed and filtered the output bam files for reads that have a soft clip (-S) followed by a match $(-\mathrm{M})$ or reads with the reverse feature $(-\mathrm{M},-\mathrm{S})$. The described features are expected from viral reads, covering the prophage attachment (attP) site in concatemeric or circularised phage genomes when mapped to the host reference genome. To determine the end position of the prophage, the reported start position of the aligned read was adjusted by adding the number of exact matched bases -1 .
The count of clipped reads, indicating the unique start and end position, was evaluated and the positions with the highest counts with the exception of start and end positions of contigs were deemed as indicative for prophage boundaries. The coordinates were additionally verified by cross-matching them to the regions of increased OPR counts.

\section{Assembly of Akkermansia muciniphila YL44 phage genomes from enriched virions}

The paired-end DNA sequencing reads from enriched virions were aligned with BWA [71] to the YL44 genome. Reads aligning to the predicted prophage regions were downsampled to $2 \times 2500$ reads and assembled with SPAdes (version 3.14) [75] using the option --isolate. Clipped read analysis to verify start and end sites of both phages was performed as described before.

\section{Estimation of genome coverage for each sample}

For the normalisation to per-cell counts, we estimated the genome coverage of each sample by calculating the median insert coverage of 10 universal single-copy marker genes (MGs) [45, 46]. For each strain, the 40 original marker genes were extracted using fetchMGs (v1.1) [45], and the following 10 MGs were subsampled (COG0012, COG0016, COG0018, COG0172, COG0215, COG0495, COG0525, COG0533, COG0541 and COG0552). For each sample, we calculated the median insert coverage of the 10 MGs using the insert coverage from featureCounts as input. The median insert coverage of the 10 MGs was used to normalise the OPRs count and to calculate $\mathrm{PtoH}$ ratios in $S$. Tm LT2 ${ }^{p 22}$.

\section{Relative abundance and coverage calculation for ex vivo community samples}

The base coverage (number of sequenced nucleotides per base of a genome) for each strain was approximated as the product of the median insert coverage of the 10 MGs and the average insert length. Subsequently, the base coverage per strain was calculated as the mean over all six (three control and three induced) replicates. The relative abundance of each strain within a community sample was calculated as the insert coverage per strain divided by the total insert coverage of all strains in the community.

\section{Verification of p22 prophage induction and comparison of PtoH ratios from qPCR and HTS experiments}

For the verification of $\mathrm{p} 22$ prophage induction, we designed qPCR primers flanking the $66 \mathrm{bp}$ long attachment sites (attL and attR) of lysogenic p22 and its host $S$. Tm $\mathrm{LT}_{2}{ }^{p 22}$. The combination of the different primers allowed for the quantification of naive $S$. Tm $\mathrm{LT}^{p 22}$ 
(Sal1: 5'-CAGGGCCGATATAGCTCAGT-3' and Sal2: 5'-TGGTGTTTTTGAGAAATGAGGTTGT-3'), lytic p22 (p1: 5'-AGGTATGACGTGGTATGTTGTTG-3' and p2: 5' -AAGGAAGGCACGAATAATACGTAAG$3^{\prime}$ ), and lysogenic $S$. Tm LT2 ${ }^{p 22}$ (Sal1 and p2) genomes. We analysed DNA of induced and control cultures of $S$. Tm LT2 ${ }^{p 22}$ with Universal FastStart SYBR Green Master (ROX; Roche) on the StepOne Plus real-time PCR system (Applied Biosystems) using the following cycling conditions: (1) initial denaturation, $95{ }^{\circ} \mathrm{C}$ for $14 \mathrm{~min}$; (2) denaturation, $94{ }^{\circ} \mathrm{C}$ for $15 \mathrm{~s}$; (3) annealing, $60{ }^{\circ} \mathrm{C}$ for $30 \mathrm{~s}$; (4) extension, $72{ }^{\circ} \mathrm{C}$ for $20 \mathrm{~s}$. Cycles $2-4$ were repeated 35 times. The prophage to host (PtoH) ratio $_{\mathrm{qPCR}}$ and $\triangle \mathrm{PtoH}$ ratio was quantified by using the formula I and III, respectively. Using HTS data, it is not possible to differentiate whether phage sequences originated from excised or inserted prophages. Similarly, bacterial sequences could originate from either naïve $S$. Tm LT2 $2^{p 22}$ or lysogenic $S$. Tm $\mathrm{LT}^{p 22}$ genomes. We thus included the $S$. Tm LT2 ${ }^{p 22}$ term in the formula to calculate $\mathrm{PtoH}$ ratio $_{\mathrm{qPCR}}$ to allow for a direct comparison to $\mathrm{PtoH}$ ratio ${ }_{\mathrm{HTS}}$, which was calculated according to formula II. For the calculation of the $\mathrm{PtoH}$ ratio ${ }_{\mathrm{HTS}}$, raw sequencing reads were processed as described above and the alignment files used as input for featureCounts to retrieve gene counts. The main difference was that the prophage sequence was used as one feature (gene) to avoid possible double-counting due to reads spanning across multiple genes. We then determined the insert coverage of the non-prophage region, approximated by the median insert coverage of the 10 MGs, and the $\mathrm{PtoH}$ ratio $_{\mathrm{HTS}}$, and the $\Delta \mathrm{PtoH}$ ratio was calculated as shown in formula II and III, respectively. We applied two statistical tests to evaluate the mean difference between $\mathrm{PtoH}$ ratio and $\Delta \mathrm{PtoH}$ ratio from qPCR and HTS experiments, one accounting for different variance between groups (Welch test) and another one assuming equal variance between groups (paired $t$ test).

I) $\mathrm{PtoH}$ ratio $_{\mathrm{qPCR}}=\left(\mathrm{p} 22+S . \mathrm{Tm} \mathrm{LT}^{p 22}\right) /($ naïve $S$. $\mathrm{Tm} \mathrm{LT}^{p 22}+S$. Tm LT2 $\left.{ }^{p 22}\right)$

II) $\mathrm{PtoH}$ ratio HTS $=$ insert coverage of $\mathrm{p} 22 /$ mean insert coverage of MGs

III) $\Delta$ PtoH ratio ${ }_{\mathrm{qPCR} / \mathrm{HTS}}=\log _{2}\left(\mathrm{PtoH}\right.$ ratio $_{\text {induced }} / \mathrm{PtoH}$ ratio $\left._{\text {control }}\right)$

For additional validation, the following $\mathrm{qPCR}$ primers pairs were used for the quantification of naïve $E$. coli $\mathrm{HS}$ (attL-f: $5^{\prime}$-TTGAGAGGGTTGCAGG GTAG-3' and attR-r: 5'-TTGTGGAGCCCATCAACC C-3'), lysogenic E. coli HS (attL-f and attL-r: 5'CCGTAATAACCACCACTGACCA-3') and lytic $E$. coli HS phage 1 (attR-f: 5'-CGCCATTTTGTCGCCA TCG-3' and attL-r) genomes.
In silico prophage prediction and comparison to experimentally validated data

In addition to the re-sequenced $S$. Tm LT2 $2^{p 22}$ genome, we used the reference genomes of the Oligo- $\mathrm{MM}^{12}$ and E. coli HS strains that were downloaded from NCBI (Additional file 2: Supplementary Table S2) for the in silico prediction of prophages using PHASTER [36], Prophage Hunter [37] ,VIBRANT [35] and VirSorter [34]. For the visual presentation and $F$-score calculation, we consider for PHASTER and Prophage Hunter prophages that were classified as complete or active and for VirSorter prediction in category one and two.

\section{Prediction of prophage replication modes by HTS data from free virions}

The replication mode of induced prophages in $\mathrm{A}$. muciniphila YL44 cultures was predicted using sequenced reads of free virions that were provided as input for PhageTerm [60]. As prophage reference genomes, we supplied the prophage sequences (Supplementary File S3) that we extracted from the host reference genome sequence using the prophage start and end coordinates determined by clipped read analysis.

\section{Cos site prediction in prophages}

Prophages that follow a unit-length packaging model initiate the packaging of viral DNA into the procapsid at the same position called the cos site. Therefore, the number of reads starting at a cos site must be increased compared to any other position in the prophage genome when the DNA originated from packed, viral DNA that was randomly sheared during library preparation. As input for the cos site prediction, we used qualitycontrolled reads, which were individually mapped as described for the OPR analysis. For the cos site identification, we filtered for properly paired reads that fell into the respective prophage regions. The start and end positions of reads were counted as described above for the clipped read analysis and putative cos sites were determined by visually inspecting sites where reads with the same start and end position accumulated within prophage regions.

\section{Supplementary Information}

The online version contains supplementary material available at https://doi. org/10.1186/s40168-021-01033-w.

Additional file 1: Supplementary Figure S1. A correct reference genome sequence is favourable for determining accurate $\Delta \mathrm{PtoH}$ ratios. Supplementary Figure S2: Several in vitro-induced Oligo- $\mathrm{MM}^{12}$ strains reveal genomic regions with putative prophages. Supplementary Figure S3. OPR analysis localtes a putative prophage region in cultured $A$. muris KB18 and F. plautii YL31. Supplementary Figure S4. Ex vivo induction of prophages in A. muciniphila YL44 and B. coccoides YL58. 
Additional file 2: Supplementary Table S1. Similar OPR counts at the attL and attR sites verify an origin of sequencing reads from circularised phage genomes. Supplementary Table S2. Collection of strains and microbiota community used in this study. Supplementary Table S3. Homologous core sequences of the attachment sites of induced prophages. Supplementary Table S4. Packaging site and its location in the lysogenic host genome.

Additional file 3. Blautia coccoides YL58 phage 1. Flavonifractor plautii YL31 phage 1. Flavonifractor plautii YL31 phage 2. Salmonella enterica serovar Typhimurium LT2 p22. Salmonella enterica serovar Typhimurium LT2 FELS-1. Salmonella enterica serovar Typhimurium LT2 FELS-2. Escherichia coli HS phage 1. Acutalibacter muris KB18 phage 1. Akkermansia muciniphila YL44 phage 1. Akkermansia muciniphila YL44 phage 1 (assembly virion enrichment). Akkermansia muciniphila YL44 phage 2. Akkermansia muciniphila YL44 phage 2 (assembly virion enrichment)

Additional file 4: Supplementary Table S5. Prophage predicted by VIBRANT. Supplementary Table S6. Prophage predicted by VirSorter. Supplementary Table S7. Prophage predicted by Prophage Hunter. Supplementary Table S8. Prophage predicted by PHASTER

\section{Acknowledgements}

We would like to thank the members of the Sunagawa, Hardt and Slack laboratories for scientific discussion, and Andrew Jermy for editing a draft version of the manuscript. Furthermore, we thank the Abram Aertsen Lab for providing the $S$. Tm $L T 2^{p 22}$ strain and Lucas Paoli for offering his computational support whenever needed. We also thank the ETH Zürich High Performance Computing facilities and the Functional Genomics Center Zurich for providing computational and sequencing support.

\section{Authors' contributions}

The study was conceived and designed by MZ, HJR and SS. The experimental work was performed by MZ with the support of NM. HJR performed the initial analysis of shotgun sequencing data and developed the mVIRs package with contributions from MC. CMF performed the sequencing, assembly and annotation of S. Tm $\mathrm{LT}^{\mathrm{p} 22}$. WDH and DH provided microbial strains and support for performing the animal experiments. MZ analysed the data and wrote the manuscript with SS and input from all other authors. The authors read and approved the final manuscript.

\section{Funding}

This project was supported by the ETH and Helmut Horten Foundation, the Novartis Foundation for Medical-Biological Research (\#17B077) and the Swiss National Science Foundation (310030_192567 to WDH and 51NF40_180575 to SS and WDH)

\section{Availability of data and materials}

The datasets generated and analysed during the current study have been deposited at the European Nucleotide Archive (ENA) with the accession no. PRJEB39818. The mVIRs (version 1.0) package for OPR detection can be found under: https://github.com/SushiLab/mVIRs. An R-Markdown for the OPR count analysis including example files is available on Zenodo https:// doi.org/10.5281/zenodo.4310165).

\section{Ethics approval and consent to participate}

All animal experiments were reviewed and approved by the Kantonales Veterinäramt, Zürich, under the licence numbers ZH193/16 and ZH158/19, and performed in compliance with the Swiss animal protection law (TschG).

\section{Consent for publication}

Not applicable for that section.

\section{Competing interests}

The authors declare that they have no competing interests.

\section{Author details}

'Department of Biology, Institute of Microbiology and Swiss Institute of Bioinformatics, ETH Zürich, Zürich, Switzerland. ²Department of Health Sciences and Technology, Institute of Food, Nutrition and Health, ETH Zürich, Zürich, Switzerland.
Received: 16 September 2020 Accepted: 9 February 2021

Published online: 29 March 2021

\section{References}

1. Virgin HW. The virome in mammalian physiology and disease. Cell. 2014; 157:142-50

2. Breitbart M, Hewson I, Felts B, Mahaffy JM, Nulton J, Salamon P, et al. Metagenomic analyses of an uncultured viral community from human feces. J Bacteriol. 2003;185:6220-3.

3. Minot S, Grunberg S, Wu GD, Lewis JD, Bushman FD. Hypervariable loci in the human gut virome. Proc Natl Acad Sci U S A. 2012;109:3962-6.

4. Reyes A, Haynes M, Hanson N, Angly FE, Heath AC, Rohwer F, et al. Viruses in the faecal microbiota of monozygotic twins and their mothers. Nature. 2010;466:334-8.

5. Shkoporov AN, Clooney AG, Sutton TDS, Ryan FJ, Daly KM, Nolan JA, et al. The human gut virome is highly diverse, stable, and individual specific. Cell Host Microbe. 2019;26:527-41.

6. Howard-Varona C, Hargreaves KR, Abedon ST, Sullivan MB. Lysogeny in nature: mechanisms, impact and ecology of temperate phages. ISME J. 2017:11:1511-20.

7. Hobbs Z, Abedon ST. Diversity of phage infection types and associated terminology: the problem with "Iytic or lysogenic". FEMS Microbiol Lett. 2016;363:fnw047.

8. Forterre P. The virocell concept and environmental microbiology. ISME J. 2013;7:233-6.

9. Shkoporov AN, Hill C. Bacteriophages of the human gut: the "known unknown" of the microbiome. Cell Host Microbe. 2019;25:195-209.

10. Warwick-Dugdale J, Buchholz HH, Allen MJ, Temperton B. Host-hijacking and planktonic piracy: how phages command the microbial high seas. Virol J. 2019;16:15.

11. Nanda AM, Thormann K, Frunzke J. Impact of spontaneous prophage induction on the fitness of bacterial populations and host-microbe interactions. J Bacteriol. 2015;197:410-9.

12. Casjens SR, Hendrix RW. Bacteriophage lambda: early pioneer and still relevant. Virology. 2015;479-480:310-30.

13. Levine M. Effect of mitomycin C on interactions between temperate phages and bacteria. Virology. 1961;13:493-9.

14. Johnson TA, Looft T, Severin AJ, Bayles DO, Nasko DJ, Wommack KE, et al. The in-feed antibiotic carbadox induces phage gene transcription in the swine gut microbiome. MBio. 2017;8:e00709-17.

15. Lwoff A. Lysogeny. Bacteriol Rev. 1953;17:269-337.

16. Hsu BB, Gibson TE, Yeliseyev V, Liu Q, Lyon L, Bry L, et al. Dynamic modulation of the gut microbiota and metabolome by bacteriophages in a mouse model. Cell Host Microbe. 2019;25:803-14.

17. Duerkop BA, Clements CV, Rollins D, Rodrigues JLM, Hooper LV. A composite bacteriophage alters colonization by an intestinal commensal bacterium. Proc Natl Acad Sci U S A. 2012;109:17621-6.

18. Norman JM, Handley SA, Baldridge MT, Droit L, Liu CY, Keller BC, et al. Disease-specific alterations in the enteric virome in inflammatory bowel disease. Cell. 2015;160:447-60.

19. Duerkop BA, Kleiner M, Paez-Espino D, Zhu W, Bushnell B, Hassell B, et al. Murine colitis reveals a disease-associated bacteriophage community. Nat Microbiol. 2018;3:1023-31.

20. Diard M, Bakkeren E, Cornuault JK, Moor K, Hausmann A, Sellin ME, et al Inflammation boosts bacteriophage transfer between salmonella spp. Science. 2017:355:1211-5.

21. Minot S, Sinha R, Chen J, Li H, Keilbaugh SA, Wu GD, et al. The human gut virome: inter-individual variation and dynamic response to diet. Genome Res. 2011;21:1616-25.

22. Howe A, Ringus DL, Williams RJ, Choo Z-N, Greenwald SM, Owens $S M$, et al. Divergent responses of viral and bacterial communities in the gut microbiome to dietary disturbances in mice. ISME J. 2016;10: $1217-27$.

23. Oh J-H, Alexander LM, Pan M, Schueler KL, Keller MP, Attie AD, et al. Dietary fructose and microbiota-derived short-chain fatty acids promote bacteriophage production in the gut symbiont lactobacillus reuteri. Cell Host Microbe. 2019;25:273-84.

24. Jiang X, Hall AB, Xavier RJ, Alm EJ. Comprehensive analysis of chromosomal mobile genetic elements in the gut microbiome reveals phylum-level niche-adaptive gene pools. PLoS One. 2019;14:e0223680. 
25. De Paepe M, Tournier L, Moncaut E, Son O, Langella P, Petit M-A. Carriage of $\lambda$ latent virus is costly for its bacterial host due to frequent reactivation in monoxenic mouse intestine. PLoS Genet. 2016;12:e1005861.

26. Calderaro A, Dettori G, Collini L, Ragni P, Grillo R, Cattani $P$, et al. Bacteriophages induced from weakly beta-haemolytic human intestinal spirochaetes by mitomycin C. J Basic Microbiol. 1998;38:323-35.

27. Reyes A, Wu M, McNulty NP, Rohwer FL, Gordon Jl. Gnotobiotic mouse model of phage-bacterial host dynamics in the human gut. Proc Natl Acad Sci U S A. 2013;110:20236-41.

28. Reyes A, Semenkovich NP, Whiteson K, Rohwer F, Gordon Jl. Going viral: next-generation sequencing applied to phage populations in the human gut. Nat Rev Microbiol. 2012;10:607-17.

29. Hertel R, Rodríguez DP, Hollensteiner J, Dietrich S, Leimbach A, Hoppert M, et al. Genome-based identification of active prophage regions by next generation sequencing in bacillus licheniformis DSM13. PLoS One. 2015;10: e0120759.

30. Hurwitz BL, Deng L, Poulos BT, Sullivan MB. Evaluation of methods to concentrate and purify ocean virus communities through comparative, replicated metagenomics. Environ Microbiol. 2013;15:1428-40.

31. Aggarwala V, Liang G, Bushman FD. Viral communities of the human gut: metagenomic analysis of composition and dynamics. Mob DNA. 2017;8:12.

32. Lasken RS, Stockwell TB. Mechanism of chimera formation during the multiple displacement amplification reaction. BMC Biotechnol. 2007;7:19.

33. Waller AS, Yamada T, Kristensen DM, Kultima JR, Sunagawa S, Koonin EV, et al. Classification and quantification of bacteriophage taxa in human gut metagenomes. ISME J. 2014;8:1391-402.

34. Roux S, Enault F, Hurwitz BL, Sullivan MB. VirSorter: mining viral signal from microbial genomic data. PeerJ. 2015;3:e985.

35. Kieft K, Zhou Z, Anantharaman KVIBRANT. Automated recovery, annotation and curation of microbial viruses, and evaluation of viral community function from genomic sequences. Microbiome. 2020;8:90.

36. Arndt D, Grant JR, Marcu A, Sajed T, Pon A, Liang Y, et al. PHASTER: a better, faster version of the PHAST phage search tool. Nucleic Acids Res. 2016;44: W16-21.

37. Song W, Sun H-X, Zhang C, Cheng L, Peng Y, Deng Z, et al. Prophage hunter: an integrative hunting tool for active prophages. Nucleic Acids Res. 2019;47:W74-80.

38. Koonin EV, Senkevich TG, Dolja W. The ancient virus world and evolution of cells. Biol Direct. 2006;1:29.

39. McClelland M, Sanderson KE, Spieth J, Clifton SW, Latreille P, Courtney L, et al. Complete genome sequence of salmonella enterica serovar Typhimurium LT2. Nature. 2001;413:852-6.

40. Sauer RT, Ross MJ, Ptashne M. Cleavage of the lambda and P22 repressors by recA protein. J Biol Chem. 1982;257:4458-62.

41. Susskind MM, Botstein D. Molecular genetics of bacteriophage P22. Microbiol Rev. 1978;42(2):385-413.

42. Gilbert W, Dressler D. DNA replication: the rolling circle model. Cold Spring Harb Symp Quant Biol. 1968;33:473-84.

43. Casjens S, Weigele P. DNA packaging by bacteriophage P22. In: Viral genome packaging machines: genetics, structure, and mechanism: Springer; 2005. p. 80-8.

44. Ramírez E, Schmidt M, Rinas U, Villaverde A. RecA-dependent viral burst in bacterial colonies during the entry into stationary phase. FEMS Microbiol Lett. 1999;170:313-7.

45. Sunagawa S, Mende DR, Zeller G, Izquierdo-Carrasco F, Berger SA, Kultima $J R$, et al. Metagenomic species profiling using universal phylogenetic marker genes. Nat Methods. 2013;10:1196-9.

46. Milanese A, Mende DR, Paoli L, Salazar G, Ruscheweyh H-J, Cuenca M, et al. Microbial abundance, activity and population genomic profiling with mOTUs2. Nat Commun. 2019;10:1014.

47. Love Ml, Huber W, Anders S. Moderated estimation of fold change and dispersion for RNA-seq data with DESeq2. Genome Biol. 2014;15:550.

48. Frye JG, Porwollik S, Blackmer F, Cheng P, McClelland M. Host gene expression changes and DNA amplification during temperate phage induction. J Bacteriol. 2005;187:1485-92.

49. Lemire S, Figueroa-Bossi N, Bossi L. Bacteriophage crosstalk: coordination of prophage induction by trans-acting antirepressors. PLoS Genet. 2011;7: e1002149.

50. Figueroa-Bossi N, Bossi L. Inducible prophages contribute to salmonella virulence in mice. Mol Microbiol. 1999;33:167-76.
51. Leong JM, Nunes-Düby S, Lesser CF, Youderian P, Susskind MM, Landy A. The phi 80 and P22 attachment sites. Primary structure and interaction with Escherichia coli integration host factor. J Biol Chem. 1985;260:4468-77.

52. Maloy S, Hughes KT, Casadesús J. The lure of bacterial genetics: a tribute to John Roth: American Society for Microbiology Press; 2010.

53. Chen J, Quiles-Puchalt N, Chiang YN, Bacigalupe R, Fillol-Salom A, Chee MSJ, et al. Genome hypermobility by lateral transduction. Science. 2018;362:207-12.

54. Casjens SR, Gilcrease EB. Determining DNA packaging strategy by analysis of the termini of the chromosomes in tailed-bacteriophage virions. Methods Mol Biol. 2009;502:91-111.

55. Sternberg N, Weisberg R. Packaging of prophage and host DNA by coliphage lambda. Nature. 1975;256:97-103.

56. Brugiroux S, Beutler M, Pfann C, Garzetti D, Ruscheweyh H-J, Ring D, et al, Genome-guided design of a defined mouse microbiota that confers colonization resistance against salmonella enterica serovar Typhimurium. Nat Microbiol. 2016;2:16215.

57. Uchimura Y, Wyss M, Brugiroux S, Limenitakis JP, Stecher B, McCoy KD, et al. Complete genome sequences of 12 species of stable defined moderately diverse mouse microbiota 2. Genome Announc. 2016;4:e00951-16.

58. Desai MS, Seekatz AM, Koropatkin NM, Kamada N, Hickey CA, Wolter M, et al. A dietary fiber-deprived gut microbiota degrades the colonic mucus barrier and enhances pathogen susceptibility. Cell. 2016:167:1339-53.e21.

59. Tenaillon O, Skurnik D, Picard B, Denamur E. The population genetics of commensal Escherichia coli. Nat Rev Microbiol. 2010;8:207-17.

60. Garneau JR, Depardieu F, Fortier L-C, Bikard D, Monot M. PhageTerm: a tool for fast and accurate determination of phage termini and packaging mechanism using next-generation sequencing data. Sci Rep. 2017;7:8292.

61. Taddei F, Matic I, Radman M. cAMP-dependent SOS induction and mutagenesis in resting bacterial populations. Proc Natl Acad Sci U S A. 1995;92:11736-40

62. Goerke C, Köller J, Wolz C. Ciprofloxacin and trimethoprim cause phage induction and virulence modulation in Staphylococcus aureus. Antimicrob Agents Chemother. 2006;50:171-7.

63. Garcia-Russell N, Elrod B, Dominguez K. Stress-induced prophage DNA replication in salmonella enterica serovar Typhimurium. Infect Genet Evol. 2009;9:889-95

64. Wozniak RAF, Waldor MK. Integrative and conjugative elements: mosaic mobile genetic elements enabling dynamic lateral gene flow. Nat Rev Microbiol. 2010;8:552-63.

65. Possoz C, Ribard C, Gagnat J, Pernodet JL, Guérineau M. The integrative element pSAM2 from Streptomyces: kinetics and mode of conjugal transfer. Mol Microbiol. 2001:42:159-66.

66. Bobay L-M, Rocha EPC, Touchon M. The adaptation of temperate bacteriophages to their host genomes. Mol Biol Evol. 2013;30:737-51.

67. Wick R. Porechop: adapter trimmer for Oxford Nanopore reads. Available from: https://github.com/rrwick/Porechop

68. Wick R. Filtlong: quality filtering tool for long reads. Available from: https:// github.com/rrwick/Filtlong

69. Kolmogorov M, Yuan J, Lin Y, Pevzner PA. Assembly of long error-prone reads using repeat graphs. Nat Biotechnol. 2019:37:540-6.

70. Walker BJ, Abeel T, Shea T, Priest M, Abouelliel A, Sakthikumar S, et al. Pilon: an integrated tool for comprehensive microbial variant detection and genome assembly improvement. PLoS One. 2014;9:e112963.

71. Li H, Durbin R. Fast and accurate short read alignment with burrowswheeler transform. Bioinformatics. 2009;25:1754-60.

72. Bushnell B. BBMap: short read aligner, and other bioinformatic tools. Available from: https://sourceforge.net/projects/bbmap/

73. Liao Y, Smyth GK, Shi W. featureCounts: an efficient general purpose program for assigning sequence reads to genomic features. Bioinformatics. 2014;30:923-30.

74. National Center for Biotechnology. NCBI. Available from: https://www.ncbi. nlm.nih.gov/genomes/GenomesGroup.cgi?taxid=10239\&host=bacteria Accessed: 29 Nov 2020.

75. Bankevich A, Nurk S, Antipov D, Gurevich AA, Dvorkin M, Kulikov AS, et al. SPAdes: a new genome assembly algorithm and its applications to singlecell sequencing. J Comput Biol. 2012;19:455-77.

\section{Publisher's Note}

Springer Nature remains neutral with regard to jurisdictional claims in published maps and institutional affiliations. 